Homology, Homotopy and Applications, vol.11(1), 2009, pp.81-114

\title{
FLAT CYCLIC FRÉCHET MODULES, AMENABLE FRÉCHET ALGEBRAS, AND APPROXIMATE IDENTITIES
}

\author{
A.YU. PIRKOVSKII
}

\author{
(communicated by Alexander Mishchenko)
}

\begin{abstract}
Let $A$ be a locally $m$-convex Fréchet algebra. We give a necessary and sufficient condition for a cyclic Fréchet $A$-module $X=A_{+} / I$ to be strictly flat, generalizing thereby a criterion of Helemskii and Sheinberg. To this end, we introduce a notion of "locally bounded approximate identity" (a locally b.a.i. for short), and we show that $X$ is strictly flat if and only if the ideal $I$ has a right locally b.a.i. Next we apply this result to amenable algebras and show that a locally $m$-convex Fréchet algebra $A$ is amenable if and only if $A$ is isomorphic to a reduced inverse limit of amenable Banach algebras. We also extend a number of characterizations of amenability obtained by Johnson and by Helemskii and Sheinberg to the setting of locally $m$-convex Fréchet algebras. As a corollary, we show that Connes and Haagerup's theorem on amenable $C^{*}$-algebras and Sheinberg's theorem on amenable uniform algebras hold in the Fréchet algebra case. We also show that a quasinormable locally $m$-convex Fréchet algebra has a locally b.a.i. if and only if it has a b.a.i. On the other hand, we give an example of a commutative, locally $m$-convex Fréchet-Montel algebra which has a locally b.a.i., but does not have a b.a.i.
\end{abstract}

\section{Introduction}

The notion of a "flat Banach module" over a Banach algebra was introduced by A.Ya. Helemskii $[\mathbf{1 7}]$ within the framework of his "Topological Homology" theory, which is a version of relative homological algebra in categories of topological modules over topological algebras (see $[\mathbf{2 0}, \mathbf{2 3}]$ ). By definition, a left Banach module $X$ over a Banach algebra $A$ is flat if the projective tensor product $(\cdot) \widehat{\otimes}_{A} X$ takes "admissible" exact sequences of right Banach $A$-modules to exact sequences of vector spaces (for

The author was partially supported by the RFBR grant 08-01-00867.

Received February 14, 2008; published on March 24, 2009.

2000 Mathematics Subject Classification: Primary 46M18, 46M10, 46H25; Secondary 16D40, 18G50, $46 \mathrm{~A} 45$.

Key words and phrases: flat Fréchet module, cyclic Fréchet module, amenable Fréchet algebra, locally $m$-convex algebra, approximate identity, approximate diagonal, Köthe space, quasinormable Fréchet space.

This article is available at http://intlpress.com/HHA/v11/n1/a5

Copyright (C) 2009, International Press. Permission to copy for private use granted. 
details, see Section 4). About the same time B.E. Johnson published his important memoir $[\mathbf{2 7}]$ where he introduced amenable Banach algebras. By definition, a Banach algebra $A$ is amenable if for each Banach $A$-bimodule $X$ every continuous derivation from $A$ to the dual bimodule, $X^{*}$, is inner. A relation between flat modules and amenable algebras was discovered by A.Ya. Helemskii and M.V. Sheinberg [18]. They proved that a Banach algebra $A$ is amenable if and only if $A_{+}$, the unitization of $A$, is a flat Banach $A$-bimodule. Using this, they gave several useful characterizations of amenability in terms of bounded approximate identities (b.a.i.'s); see Theorem 1.2 below. These characterizations were obtained as a consequence of the following flatness criterion for cyclic Banach modules $[\mathbf{1 7}, \mathbf{1 8}]$ (for terminology, see Section 4).

Theorem 1.1 (Helemskii, Sheinberg). Let $A$ be a Banach algebra, and let $I \subset A_{+}$be a closed left ideal. Then the following conditions are equivalent:

(i) $A_{+} / I$ is strictly flat;

(ii) I has a right b.a.i.

If, in addition, $I$ is weakly complemented in $A_{+}$(i.e., if the annihilator of $I$ is complemented in $A_{+}^{*}$ ), then (i) and (ii) are equivalent to:

(iii) $A_{+} / I$ is flat.

In order to formulate the above-mentioned characterizations of amenability obtained by Helemskii and Sheinberg, let us introduce some notation. Given a Banach algebra $A$, set $A^{e}=A_{+} \widehat{\otimes} A_{+}^{\mathrm{op}}$ (respectively, $A_{0}^{e}=A \widehat{\otimes} A^{\mathrm{op}}$ ), where $A^{\mathrm{op}}$ is the algebra opposite to $A$, and let $I^{\Delta}$ (respectively, $I_{0}^{\Delta}$ ) denote the kernel of the product map $A^{e} \rightarrow A_{+}$(respectively, $A_{0}^{e} \rightarrow A$ ).

Theorem 1.2 (Helemskii, Sheinberg). Let A be a Banach algebra. The following conditions are equivalent:

(i) $A$ is amenable;

(ii) $A_{+}$is a strictly flat Banach A-bimodule;

(iii) $A$ is biflat and has a b.a.i.;

(iv) $I^{\Delta}$ has a right b.a.i.;

(v) A has a b.a.i., and $I_{0}^{\Delta}$ has a right b.a.i.

Condition (v) of Theorem 1.2 is similar in spirit to an earlier result of Johnson [28] (for terminology, see Section 9).

Theorem 1.3 (Johnson). Let $A$ be a Banach algebra. The following conditions are equivalent:

(i) $A$ is amenable;

(ii) A has a bounded approximate diagonal;

(iii) A has a virtual diagonal.

The aim of the present paper is to generalize Theorems 1.1, 1.2, and 1.3 to locally $m$-convex Fréchet algebras (i.e., to Fréchet-Arens-Michael algebras, in the terminology of [21]). Let us remark that the question of whether Theorem 1.2 holds for nonnormable locally convex algebras was posed explicitly in [22]. The basic difficulty here 
is that the duality between flat and injective Banach modules, which was heavily used by Helemskii and Sheinberg, is not available within the framework of Fréchet modules. In fact, given a Fréchet module over a Fréchet algebra $A$, the dual module, $X^{*}$, has no reasonable topology making it into a Fréchet space. Moreover, the action of $A$ on $X^{*}$ often fails to be jointly continuous with respect to any "natural" topology on $X^{*}$ (cf. [55]). Let us also remark that many Fréchet algebras do not have nontrivial injective Fréchet modules at all $[\mathbf{4 2}, \mathbf{4 3}, 44]$.

To overcome this difficulty, we use the Arens-Michael decomposition for complete locally $m$-convex algebras $[\mathbf{3}, \mathbf{3 6}]$ and for complete locally convex modules over such algebras [46]. It turns out, however, that the property of having a bounded approximate identity does not behave well under the Arens-Michael decomposition. That is why we have to introduce a new notion of "locally bounded approximate identity", which might be of independent interest.

The paper is organized as follows. Section 2 contains some background material on topological algebras and modules. In Section 3 we prove some general results on the behavior of the projective tensor product functor. In particular, we show that this functor is cokernel-preserving. In Section 4 we discuss flat and strictly flat Fréchet modules, and we show that a (strictly) flat Banach module over a Banach algebra remains (strictly) flat when considered as a Fréchet module. In Section 5 we prove some general facts on approximate identities and approximate diagonals in topological algebras. Although these facts are well known in the case of Banach algebras, we give full proofs for the sake of completeness. In Section 6 we introduce locally bounded approximate identities and locally bounded approximate diagonals and study their basic properties. Section 7 contains the main result of the paper (Theorem 7.2 and Corollary 7.3), which extends the first part of Helemskii and Sheinberg's Theorem 1.1 (specifically, the equivalence of (i) and (ii)) to Fréchet-Arens-Michael algebras. In Section 8, by using a result of Palamodov [38], we show that for quasinormable Fréchet-Arens-Michael algebras the notions of "locally bounded" and "bounded" approximate identities are equivalent. In Section 9 we extend the second part of Helemskii and Sheinberg's Theorem 1.1 (specifically, the equivalence of (i) and (iii)) to Fréchet-Arens-Michael algebras, and then apply this result to characterizing amenability for such algebras, in the spirit of Theorems 1.2 and 1.3. In particular, we obtain a partial answer to a question posed by Helemskii [22, Problem 11]. We also prove that a Fréchet-Arens-Michael algebra is amenable if and only if it is isomorphic to a reduced inverse limit of amenable Banach algebras. This result is then used to show that a $\sigma-C^{*}$-algebra is amenable if and only if it is nuclear, and that a uniform Fréchet algebra is amenable if and only if it is isomorphic to the algebra of continuous functions on a hemicompact $k$-space. Finally, in Section 10 we give an example of a (non-quasinormable) commutative FréchetArens-Michael algebra (which is, in addition, a Montel space) with a locally b.a.i., but without a b.a.i. This example shows that Helemskii and Sheinberg's Theorem 1.1 does not extend verbatim to locally $m$-convex Fréchet algebras.

Some of the results of this paper were announced in [45]. A related result was independently obtained by C.P. Podara [47]. Specifically, she proves that implication (ii) $\Rightarrow$ (i) of Helemskii and Sheinberg's Theorem 1.1 holds for every (not necessarily locally $m$-convex) Fréchet algebra, and that condition (ii) implies also that $I$ is strictly flat. 


\section{Preliminaries}

We shall work over the complex numbers $\mathbb{C}$. Let Vect denote the category of all vector spaces and linear maps. The category of locally convex spaces (l.c.s.'s) and continuous linear maps will be denoted by LCS. Let us remark that we do not require locally convex spaces to be Hausdorff, but we follow the convention that complete l.c.s.'s are Hausdorff by definition. The full subcategories of LCS consisting of complete l.c.s.'s (respectively, of Fréchet spaces, of Banach spaces) will be denoted by $\mathrm{LCS}^{\wedge}$ (respectively, Fr, Ban).

The completion of an l.c.s. $E$ is defined to be the completion of the associated Hausdorff l.c.s., $E / \overline{\{0\}}$, and is denoted by $E^{\sim}$. We denote by $E^{*}$ the dual of $E$ and always endow $E^{*}$ with the strong topology, unless stated otherwise. The canonical embedding of $E$ into $E^{* *}$ is denoted by $i_{E}$. The space of all continuous linear maps between l.c.s.'s $E$ and $F$ is denoted by $\mathscr{L}(E, F)$. Given l.c.s.'s $E, F, G$, we denote by $\mathscr{B i l}(E \times F, G)$ the space of all jointly continuous bilinear maps from $E \times F$ to $G$. The completed projective tensor product of $E$ and $F$ is denoted by $E \widehat{\otimes} F$.

By a topological algebra we mean a topological vector space $A$ together with the structure of an associative algebra such that the product map $A \times A \rightarrow A$ is separately continuous. In what follows, when using the word "algebra" with an adjective that describes a linear topological property (such as "locally convex", "complete", "Fréchet" etc.), we mean that the underlying topological vector space of the algebra in question has the specified property. The same applies to topological modules (see below). A complete locally convex algebra with jointly continuous product is called a $\widehat{\otimes}$-algebra $[\mathbf{2 0 , 5 5}$. A seminorm $\|\cdot\|$ on an algebra $A$ is submultiplicative if $\|a b\| \leqslant\|a\|\|b\|$ for all $a, b \in A$. A locally convex algebra $A$ is locally $m$-convex [36] if the topology on $A$ can be defined by a family of submultiplicative seminorms. Equivalently, $A$ is locally $m$-convex if it has a base $\mathscr{U}$ of 0 -neighborhoods consisting of absolutely convex, idempotent sets (here "idempotent" means that $U^{2} \subset U$ for each $U \in \mathscr{U})$. An Arens-Michael algebra [21] is a complete locally $m$-convex algebra. A Fréchet-Arens-Michael algebra is a locally $m$-convex Fréchet algebra.

Let $A$ be a topological algebra. Following [26], we say that $A$ is left hypotopological if the product $A \times A \rightarrow A$ is left hypocontinuous with respect to the family of all bounded subsets of $A$. This means that for each 0 -neighborhood $V \subset A$ and each bounded subset $B \subset A$ there exists a 0-neighborhood $U \subset A$ such that $U B \subset V$. Right hypotopological algebras are defined similarly. "Hypotopological" means "left and right hypotopological". It is easy to show (cf. [26]) that the product of two bounded sets in a left hypotopological algebra is bounded. Indeed, given bounded sets $B_{1}, B_{2} \subset A$ and a 0 -neighborhood $U \subset A$, we may successively find a 0-neighborhood $V \subset A$ such that $V B_{2} \subset U$ and $\lambda>0$ such that $B_{1} \subset \lambda V$. Then $B_{1} B_{2} \subset \lambda U$, so that $B_{1} B_{2}$ is bounded. The same argument works in the case of a right hypotopological algebra.

If $A$ is a left hypotopological locally convex algebra, then the strong bidual, $A^{* *}$, can be endowed with a product making it into a topological algebra [16]. The details of the construction are similar to the Banach algebra case (see, e.g., [6, 2.6], [39, 1.4]). Specifically, given $f \in A^{*}$ and $a \in A$, define $f \cdot a \in A^{*}$ by $\langle f \cdot a, b\rangle=\langle f, a b\rangle(b \in A)$. Next, given $u \in A^{* *}$ and $f \in A^{*}$, define $u \cdot f \in A^{*}$ by $\langle u \cdot f, a\rangle=\langle u, f \cdot a\rangle(a \in A)$. Finally, given $u, v \in A^{* *}$, define $u v \in A^{* *}$ by $\langle u v, f\rangle=\langle u, v \cdot f\rangle\left(f \in A^{*}\right)$. The bilinear map 
$(u, v) \mapsto u v$ is a separately continuous, associative product on $A^{* *}$ (the first Arens product) $[\mathbf{1 6}, 3.8,3.9]$. The canonical embedding $i_{A}: A \rightarrow A^{* *}$ is an algebra homomorphism $[\mathbf{1 6}, 3.5]$. The first Arens product is weak ${ }^{*}$-weak ${ }^{*}$ continuous with respect to the first variable, i.e., for each $v \in A^{* *}$ the map $A^{* *} \rightarrow A^{* *}, u \mapsto u v$, is continuous with respect to the weak* topology on both copies of $A^{* *}[\mathbf{1 6}, 3.4]$. In general, the first Arens product does not have the above property with respect to the second variable. However, for each $a \in A$ the map $A^{* *} \rightarrow A^{* *}, v \mapsto i_{A}(a) v$ is continuous with respect to the weak* topology on both copies of $A^{* *}[\mathbf{1 6}, 3.6]$.

Given a topological algebra $A$, a left semitopological $A$-module is a topological vector space $X$ together with the structure of a left $A$-module such that for each $a \in A$ the map $X \rightarrow X, x \mapsto a \cdot x$ is continuous. If, in addition, for each $x \in X$ the map $A \rightarrow X, a \mapsto a \cdot x$ is continuous, then we say that $X$ is a left topological $A$-module. Right (semi)topological $A$-modules and (semi)topological $A$-bimodules are defined similarly. In what follows, "locally convex $A$-module" means "locally convex topological $A$-module". If $A$ is a $\widehat{\otimes}$-algebra and $X$ is a left locally convex $A$-module, we say that $X$ is a left $A-\widehat{\otimes}$-module $[\mathbf{2 0}, \mathbf{5 5}]$ if $X$ is complete and the action $A \times X \rightarrow X$ is jointly continuous. If $X$ and $Y$ are left semitopological $A$-modules (respectively, right semitopological $A$-modules, semitopological $A$-bimodules), then the space of all continuous left $A$-module morphisms (respectively, right $A$-module morphisms, $A$-bimodule morphisms) from $X$ to $Y$ is denoted by ${ }_{A} \mathbf{h}(X, Y)$ (respectively, $\mathbf{h}_{A}(X, Y),{ }_{A} \mathbf{h}_{A}(X, Y)$ ).

If $A$ is a locally convex algebra and $X$ is a left semitopological locally convex $A$ module, then $X^{*}$ is a right semitopological locally convex $A$-module with respect to the action $\langle f \cdot a, x\rangle=\langle f, a \cdot x\rangle\left(f \in X^{*}, a \in A, x \in X\right)$. Similarly, if $X$ is a right semitopological locally convex $A$-module, then $X^{*}$ is a left semitopological locally convex $A$-module. If the action of $A$ on $X$ is hypocontinuous with respect to the family of all bounded subsets of $X$, then the action of $A$ on $X^{*}$ is separately continuous, so that $X^{*}$ is a locally convex $A$-module (cf. $[\mathbf{1 6}, 3.1]$ or $[\mathbf{5 5}$, Section 3$]$ ). Note that the above condition is satisfied automatically whenever $A$ is barrelled and $X$ is a locally convex $A$-module.

Let $A$ be an algebra, and let $A_{+}$denote the unitization of $A$. We denote by $j_{A}$ the embedding of $A$ into $A_{+}$, and by $\varepsilon_{A}: A_{+} \rightarrow \mathbb{C}$ the homomorphism that vanishes on $A$ and satisfies $\varepsilon_{A}\left(1_{+}\right)=1$, where $1_{+}$is the identity of $A_{+}$. Each algebra homomorphism $\varphi: A \rightarrow B$ uniquely extends to a unital homomorphism $\varphi_{+}: A_{+} \rightarrow B_{+}$. The one-dimensional left (respectively, right) $A$-module $A_{+} / A$ will be denoted by $\mathbb{C}_{\ell}$ (respectively, $\mathbb{C}_{r}$ ). Note that $\mathbb{C}_{\ell}^{*}$ is isomorphic to $\mathbb{C}_{r}$, and vice versa.

The right annihilator of $A$ is

$$
\operatorname{rann}(A)=\{b \in A ; a b=0 \forall a \in A\} .
$$

If $A$ is a left hypotopological algebra, then it readily follows from the definition of the first Arens product that

$$
\operatorname{rann}\left(A^{* *}\right)=\mathbf{h}_{A}\left(A^{*}, \mathbb{C}_{r}\right) .
$$

Let $A$ be a locally convex algebra, and let $A$-lcmod denote the category of all left locally convex $A$-modules. Clearly, $A$-Icmod is an additive category. For our purposes, it is important that $A$-lcmod admits inverse and direct limits. Indeed, if $\mathscr{X}=$ $\left(X_{\alpha}, \varphi_{\alpha \beta}\right)_{\alpha \in \Lambda}$ is a direct system in $A$-Icmod (i.e., a covariant functor from a directed 
set $\Lambda$ to $A$-lcmod), then the direct limit of $\mathscr{X}$ taken in the category of all left $A$ modules and endowed with the locally convex direct limit topology is easily seen to be a direct limit of $\mathscr{X}$ in $A$-lcmod. A similar argument works for inverse limits.

If $A$ is a $\widehat{\otimes}$-algebra, then the full subcategory of $A$-lcmod consisting of $\widehat{\otimes}$-modules will be denoted by $A$-mod. Given a full additive subcategory $\mathscr{C}$ of LCS , we denote by $A$-mod$(\mathscr{C})$ the full subcategory of $A$-mod consisting of the modules whose underlying l.c.s.'s are objects of $\mathscr{C}$. The respective categories of right modules and bimodules will be denoted by $\bmod -A(\mathscr{C})$ and $A$-mod- $A(\mathscr{C})$ (or just by $\bmod -A$ and $A$-mod- $A$ in the case where $\mathscr{C}=\mathrm{LCS}^{\widehat{ }}$ ). If $A$ is unital, then $A$-unmod $(\mathscr{C})$ (respectively unmod- $A(\mathscr{C}), A$-unmod- $A(\mathscr{C})$ ) stands for the full subcategory of $A$-mod $(\mathscr{C})$ (respectively of mod- $A(\mathscr{C}), A$-mod- $A(\mathscr{C})$ ) consisting of unital modules. Let us remark that $A-\bmod (\mathscr{C})$ is isomorphic to $A_{+}-\operatorname{unmod}(\mathscr{C})$, and $A-\bmod -A(\mathscr{C})$ is isomorphic to $A^{e}$-unmod $(\mathscr{C})$, where $A^{e}=A_{+} \widehat{\otimes} A_{+}^{\text {op }}$ is the enveloping algebra of $A$ [20, II.5.11]. Note also that inverse limits exist in $A$-mod and coincide with inverse limits taken in $A$-lcmod.

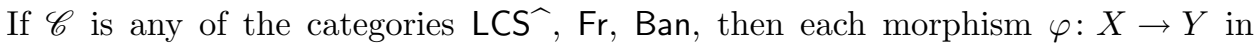
$A-\bmod (\mathscr{C})$ has a kernel and a cokernel. Specifically, $\operatorname{Ker} \varphi=\varphi^{-1}(0)$ endowed with the relative topology, and $\operatorname{Coker} \varphi=(Y / \overline{\varphi(X)})^{\sim}$, where $Y \overline{\varphi(X)}$ is endowed with the quotient topology. The morphism $\operatorname{ker} \varphi$ is just the embedding of $\varphi^{-1}(0)$ into $X$, and coker $\varphi$ is the composition of the quotient map $Y \rightarrow Y / \overline{\varphi(X)}$ with the embedding of the target into its completion. Note that if $\mathscr{C}$ is either $\operatorname{Fr}$ or Ban, then $Y / \overline{\varphi(X)}$ is already complete. A morphism $\varphi: X \rightarrow Y$ is a kernel (i.e., there exists a morphism $\psi: Y \rightarrow Z$ such that $\varphi=\operatorname{ker} \psi$ ) if and only if $\varphi$ is topologically injective, i.e., is a homeomorphism onto $\varphi(X)$. Similarly, $\varphi: X \rightarrow Y$ is a cokernel if and only if $\varphi$ is an open map onto a dense submodule of $Y$. If $\mathscr{C}$ is either Fr or Ban, then the Open Mapping Theorem implies that $\varphi$ is a kernel (respectively, a cokernel) if and only if it is injective with closed range (respectively, if and only if it is onto). For $A=0$ (i.e., in the case where $A-\bmod (\mathscr{C})=\mathscr{C})$, the above facts can be found in $[48$, Section 3], and the same argument works for any $\widehat{\otimes}$-algebra $A$.

Let $E$ be a vector space, and let $p$ be a seminorm on $E$. We denote by $E_{p}$ the completion of $E$ with respect to $p$, and by $\tau_{p}$ the canonical map $E \rightarrow E_{p}$. Note that $\operatorname{Ker} \tau_{p}=p^{-1}(0)$. If $U \subset E$ is an absorbent, absolutely convex set with Minkowski functional $p_{U}$, we write $E_{U}$ for $E_{p_{U}}$ and $\tau_{U}$ for $\tau_{p_{U}}$. Suppose that $E$ carries a locally convex topology determined by a directed family of seminorms $\left\{p_{\lambda}=\|\cdot\|_{\lambda}: \lambda \in \Lambda\right\}$. For each $\lambda \in \Lambda$, set $E_{\lambda}=E_{p_{\lambda}}$ and $\tau_{\lambda}=\tau_{p_{\lambda}}$. Given $\lambda, \mu \in \Lambda$, we write $\lambda \prec \mu$ if $\|x\|_{\lambda} \leqslant\|x\|_{\mu}$ for all $x \in E$. If $\lambda, \mu \in \Lambda$ and $\lambda \prec \mu$, then there is a unique continuous linear map $\tau_{\lambda}^{\mu}: E_{\mu} \rightarrow E_{\lambda}$ such that $\tau_{\lambda}=\tau_{\lambda}^{\mu} \tau_{\mu}$. The family $\left\{\tau_{\lambda}: \lambda \in \Lambda\right\}$ determines a continuous linear map from $E$ to the inverse $\operatorname{limit} \underset{\lim }{\longleftarrow}\left(E_{\lambda}, \tau_{\lambda}^{\mu}\right)$, which, in turn, yields a topological isomorphism between $E^{\sim}$ and $\lim \left(E_{\lambda}, \overleftarrow{\tau_{\lambda}^{\mu}}\right.$ ) (see, e.g., [51, II.5.4]). Note that the inverse system $\left(E_{\lambda}, \tau_{\lambda}^{\mu}\right)$ is reduced, i.e., for each $\mu \in \Lambda$ the canonical map $\lim _{\lambda} E_{\lambda} \rightarrow E_{\mu}$ has dense range. Conversely, if a complete locally convex space $E$ is isomorphic to a reduced inverse limit $\lim \left(E_{\lambda}, \tau_{\lambda}^{\mu}\right)$ of Banach spaces, then the topology on $E$ is determined by the family of seminorms $\left\{p_{\lambda}=\left\|\tau_{\lambda}(\cdot)\right\|: \lambda \in \Lambda\right\}$, where $\tau_{\lambda}: E \rightarrow E_{\lambda}$ is the canonical map, and the inverse system $\left(E_{\lambda}, \tau_{\lambda}^{\mu}\right)$ is isomorphic to the system constructed from the family $\left\{p_{\lambda}\right\}$ as described above. 
If $A$ is an algebra and $p$ is a submultiplicative seminorm on $A$, then $A_{p}$ is a Banach algebra and $\tau_{p}$ is an algebra homomorphism. Therefore, if $A$ is an Arens-Michael algebra with topology determined by a directed family $\left\{\|\cdot\|_{\lambda}: \lambda \in \Lambda\right\}$ of submultiplicative seminorms, then all the maps $\tau_{\lambda}$ and $\tau_{\lambda}^{\mu}$ are continuous algebra homomorphisms, and so we obtain a topological algebra isomorphism $A \cong \lim A_{\lambda}$ (the Arens-Michael decomposition theorem). The situation described above is usually expressed by the phrase "Let $A$ be an Arens-Michael algebra, and let $A=\lim A_{\lambda}$ be an Arens-Michael decomposition of $A$ ".

If $A$ is a locally convex algebra and $X$ is a left locally convex $A$-module, then we say that a continuous seminorm $q$ on $X$ is $m$-compatible if there exists a continuous submultiplicative seminorm $p$ on $A$ such that $q(a \cdot x) \leqslant p(a) q(x)$. In this case, $X_{q}$ is a Banach $A_{p}$-module (and hence a Banach $A$-module) in a natural way, and $\tau_{q}: X \rightarrow X_{q}$ is an $A$-module morphism. If $A$ is an Arens-Michael algebra and $X$ is a left $A$ - $\widehat{\otimes}$ module, then the topology on $X$ can be determined by a directed family $\left\{\|\cdot\|_{\lambda}: \lambda \in \Lambda\right\}$ of $m$-compatible seminorms $[46,3.4]$. Now the maps $\tau_{\lambda}$ and $\tau_{\lambda}^{\mu}$ are morphisms in $A$-mod, and so $X$ is isomorphic to $\lim _{\lambda} X_{\lambda}$ in $A$-mod. As above, we express this situation by the phrase "Let $A$ be an Arens-Michael algebra, let $X$ be a left $A-\widehat{\otimes}-$ module, and let $X=\lim X_{\lambda}$ be an Arens-Michael decomposition of $X$ ".

We end this section by recalling a result of Palamodov on the vanishing of the derived inverse limit functor $([\mathbf{3 7}, \mathbf{3 8}]$; see also $[\mathbf{5 6}$, Chapter 3$])$. The theorem below is a special case of [38, Corollary 5.1]; cf. also [1, Theorems 4 and 16] and [2, Theorem $2.5]$.

Theorem 2.1 (Palamodov). Let $\mathscr{X}=\left(X_{n}, \varphi_{n}^{m}\right)_{n \in \mathbb{N}}$ be an inverse sequence of Banach spaces with linking maps $\varphi_{n}^{m}: X_{m} \rightarrow X_{n}(n \leqslant m)$. Then the following conditions are equivalent:

(i) $\lim ^{1} \mathscr{X}=0$;

(ii) for each $n \in \mathbb{N}$ there exists $m \geqslant n$ such that for each $k \geqslant m$ the subspace $\varphi_{n}^{k}\left(X_{k}\right)$ is dense in $\varphi_{n}^{m}\left(X_{m}\right)$.

\section{Some properties of the projective tensor product}

Let $A$ be an algebra, $X$ a right $A$-module, $Y$ a left $A$-module, and $E$ a vector space. Recall that a bilinear map $\Phi: X \times Y \rightarrow E$ is $A$-bilinear (or balanced) if $\Phi(x \cdot a, y)=$ $\Phi(x, a \cdot y)$ for all $x \in X, y \in Y, a \in A$. If $A$ is a topological algebra, $X, Y$ are topological $A$-modules, and $E$ is a topological vector space, then the space of all jointly continuous $A$-bilinear maps from $X \times Y$ to $E$ will be denoted by $\mathscr{B} i l_{A}(X \times Y, E)$.

Now suppose that $A$ is a $\widehat{\otimes}$-algebra, $X, Y$ are $\widehat{\otimes}$-modules, and $E$ is a complete l.c.s. Then there exists a natural vector space isomorphism

$$
\mathscr{B i l}_{A}(X \times Y, E) \cong \mathscr{L}(X \underset{A}{\widehat{\otimes}} Y, E),
$$

where $X \widehat{\otimes}_{A} Y$ is the $A$-module projective tensor product of $X$ and $Y$ [20, II.4]. By definition, $X \widehat{\otimes}_{A} Y=(X \widehat{\otimes} Y / N)^{\sim}$, where $N \subset X \widehat{\otimes} Y$ is the closure of the linear span of all elements of the form $x \cdot a \otimes y-x \otimes a \cdot y \quad(x \in X, y \in Y, a \in A)$. 
The following two propositions are easy generalizations of the corresponding results on Banach modules (see [20, II.3.17] and [20, II.5.21], respectively). For Fréchet modules, Proposition 3.1 was also proved in $[\mathbf{4 7}]$.

Proposition 3.1. Let $A$ be a $\widehat{\otimes}$-algebra, and let $I \subset A_{+}$be a closed left ideal. Then for each $X \in \bmod -A$ there is a topological isomorphism

$$
X \underset{A}{\widehat{\otimes}}\left(A_{+} / I\right)^{\sim} \cong(X / \overline{X \cdot I})^{\sim}
$$

uniquely determined by $x \otimes(a+I) \mapsto x \cdot a+\overline{X \cdot I}$.

Proof. For each complete l.c.s. E, we have natural isomorphisms

$$
\begin{aligned}
\mathscr{L}\left(X \widehat{\otimes}_{A}\left(A_{+} / I\right)^{\sim}, E\right) & \cong \mathscr{B} i l_{A}\left(X \times\left(A_{+} / I\right)^{\sim}, E\right) \\
& \cong \mathscr{B} i l_{A}\left(X \times\left(A_{+} / I\right), E\right) \\
& \cong\left\{\Phi \in \mathscr{B} i l_{A}\left(X \times A_{+}, E\right) ;\left.\Phi\right|_{X \times I}=0\right\} \\
& \cong\left\{\varphi \in \mathscr{L}(X, E) ;\left.\varphi\right|_{X \cdot I}=0\right\} \\
& \cong \mathscr{L}\left((X / \overline{X \cdot I})^{\sim}, E\right) .
\end{aligned}
$$

Setting $E=(X / \overline{X \cdot I})^{\sim}$ and applying the above to the identity map of $E$, we get the result.

Proposition 3.2. Let $A$ be a $\widehat{\otimes}$-algebra, $X$ a right $A$ - $\widehat{\otimes}$-module, and $Y$ a left $A-\widehat{\otimes}$ module. There is a natural linear map

$$
(X \underset{A}{\widehat{\otimes}} Y)^{*} \rightarrow \mathbf{h}_{A}\left(X, Y^{*}\right), \quad f \mapsto(x \mapsto(y \mapsto f(x \otimes y))) .
$$

The above map is a vector space isomorphism in either of the following cases:

(i) $Y$ is a Banach A-module;

(ii) both $X$ and $Y$ are Fréchet A-modules.

Proof. Clearly, (2) is injective. Given $\varphi \in \mathbf{h}_{A}\left(X, Y^{*}\right)$, we define an $A$-bilinear map $\Phi: X \times Y \rightarrow \mathbb{C}$ by $\Phi(x, y)=\varphi(x)(y)$. It is easy to see that $\Phi$ is separately continuous, and, moreover, is hypocontinuous with respect to the family of all bounded subsets of $Y$. In either of cases (i) and (ii) this implies that $\Phi$ is jointly continuous (case (i) is obvious, while case (ii) follows from [51, III.5.1]). Therefore there exists $f \in\left(X \widehat{\otimes}_{A} Y\right)^{*}$ such that $f(x \otimes y)=\Phi(x, y)$ for all $x \in X, y \in Y$. Thus (2) is onto, as required.

The projective tensor product preserves cokernels (cf. [32, V.5.1]).

Proposition 3.3. Let $A$ be a $\widehat{\otimes}$-algebra, and let

$$
X_{1} \stackrel{f}{\rightarrow} X_{2} \stackrel{g}{\rightarrow} X_{3} \rightarrow 0
$$

be a sequence in mod- $A$ such that $g=$ coker $f$. Take $Y \in A$-mod, and consider the sequence

$$
X_{1} \widehat{\otimes} Y \stackrel{f \otimes 1_{Y}}{\longrightarrow} X_{2} \underset{A}{\widehat{\otimes}} Y \stackrel{g \otimes 1_{Y}}{\longrightarrow} X_{3} \underset{A}{\widehat{\otimes}} Y \rightarrow 0 .
$$

Then $g \otimes 1_{Y}=\operatorname{coker}\left(f \otimes 1_{Y}\right)$ in $\mathrm{LCS}^{\widehat{L}}$. 
Proof. We have to show that for each complete l.c.s. E, the sequence

$$
0 \rightarrow \mathscr{L}\left(X_{3} \underset{A}{\widehat{\otimes}} Y, E\right) \rightarrow \mathscr{L}\left(X_{2} \underset{A}{\widehat{\otimes}} Y, E\right) \rightarrow \mathscr{L}\left(X_{1} \underset{A}{\widehat{\otimes}} Y, E\right)
$$

is exact in Vect. By the definition of the projective tensor product, the latter sequence can be identified with

$$
0 \rightarrow \mathscr{B} i l_{A}\left(X_{3} \times Y, E\right) \stackrel{\alpha}{\rightarrow} \mathscr{B} i l_{A}\left(X_{2} \times Y, E\right) \stackrel{\beta}{\rightarrow} \mathscr{B} i l_{A}\left(X_{1} \times Y, E\right),
$$

where $\alpha$ and $\beta$ are induced by $g$ and $f$ in the obvious way. Since $g\left(X_{2}\right)$ is dense in $X_{3}$, we see that $\operatorname{Ker} \alpha=0$. Now suppose that $\Phi \in \operatorname{Ker} \beta$, i.e., that $\Phi(x, y)=0$ for all $x \in f\left(X_{1}\right)$ and all $y \in Y$. Since $f\left(X_{1}\right)$ is dense in $\operatorname{Ker} g$, we have $\Phi(x, y)=0$ for all $x \in \operatorname{Ker} g$. Therefore there exists a unique $A$-bilinear map $\Psi_{0}: g\left(X_{2}\right) \times Y \rightarrow E$ such that $\Psi_{0}(g(x), y)=\Phi(x, y)$ for all $x \in X_{2}, y \in Y$. Since $g: X_{2} \rightarrow g\left(X_{2}\right)$ is open, we see that $\Psi_{0}$ is jointly continuous. As $g\left(X_{2}\right)$ is dense in $X_{3}$, this implies that $\Psi_{0}$ uniquely extends to a map $\Psi \in \mathscr{B}_{i} l_{A}\left(X_{3} \times Y, E\right)$. Clearly, $\alpha(\Psi)=\Phi$. Thus (3) is exact, and so $g \otimes 1_{Y}=\operatorname{coker}\left(f \otimes 1_{Y}\right)$, as required.

Remark 3.4. In the setting of Banach modules over Banach algebras, Proposition 3.3 becomes an easy consequence of $[\mathbf{3 3}, \mathrm{V} .5]$. Indeed, in this case the functor $(\cdot) \widehat{\otimes}_{A} Y$ has a right adjoint (namely, $\mathscr{L}(Y, \cdot))$ and therefore it preserves colimits.

\section{Flat and strictly flat Fréchet modules}

Let $\mathscr{A}$ be an additive category with kernels and cokernels. An exact pair in $\mathscr{A}$ is a sequence $X \stackrel{i}{\rightarrow} Y \stackrel{p}{\rightarrow} Z$ such that $i=\operatorname{ker} p$ and $p=$ coker $i$.

Remark 4.1. It is elementary to check that $X \stackrel{i}{\rightarrow} Y \stackrel{p}{\rightarrow} Z$ is an exact pair if and only if $p=\operatorname{coker} i$ and $i$ is a kernel, or, equivalently, if and only if $i=\operatorname{ker} p$ and $p$ is a cokernel.

By a pre-exact category, we mean an additive category $\mathscr{A}$ endowed with a class $\mathscr{E}$ of exact pairs closed under isomorphism. Exact pairs belonging to $\mathscr{E}$ will be called admissible pairs or short admissible sequences. A morphism $i: X \rightarrow Y$ (respectively a morphism $p: Y \rightarrow Z$ ) is an admissible monomorphism (respectively, an admissible epimorphism) if it fits into an admissible pair $X \stackrel{i}{\rightarrow} Y \stackrel{p}{\rightarrow} Z$. A chain complex $C_{\bullet}=$ $\left(C_{n}, d_{n}\right)_{n \in \mathbb{Z}}$ in $\mathscr{A}$ is admissible if for each $n \in \mathbb{Z}$ the morphism $d_{n}: C_{n} \rightarrow C_{n-1}$ has a kernel, and the sequence $\operatorname{Ker} d_{n} \rightarrow C_{n} \rightarrow \operatorname{Ker} d_{n-1}$ is admissible.

Example 4.2. Each additive category $\mathscr{A}$ becomes a pre-exact category if we define $\mathscr{E}$ to be the class of all exact pairs in $\mathscr{A}$. In this case we say that $\mathscr{A}$ is endowed with the strongest pre-exact structure. In the sequel, the categories Vect, LCS, LCS ${ }^{\wedge}, \mathrm{Fr}$, and Ban will be considered as pre-exact categories with respect to the strongest pre-exact structure. If $A$ is a $\widehat{\otimes}$-algebra and $\mathscr{C} \subset \mathrm{LCS}^{\wedge}$ is a full additive subcategory, then the category $A-\bmod (\mathscr{C})$ endowed with the strongest pre-exact structure will be denoted by $A$-mod$(\mathscr{C})$. The strongest pre-exact categories $\bmod A(\mathscr{C})$ and $A$-mod $A(\mathscr{C})$ (as well as $A$-unmod $(\mathscr{C})$, unmod $A(\mathscr{C})$ and $A$ unmod $A(\mathscr{C})$ for $A$ unital) are defined similarly. Note that if $\mathscr{C}$ is either Ban or Fr, then a chain complex in any of the above categories is admissible if and only if it is (algebraically) exact. 
Remark 4.3. As was observed in [48], the categories $\mathscr{C}=\mathrm{LCS}, \mathscr{C}=\mathrm{Fr}$, and $\mathscr{C}=$ Ban are in fact exact categories in the sense of Quillen [49], but this is not the case for $\mathscr{C}=\mathrm{LCS}^{\wedge}$. Similar statements hold for the respective $A$-module categories $A$-mod $(\mathscr{C})$, $\underline{\bmod } A(\mathscr{C}), A-\bmod A(\mathscr{C})$. This is the reason why we have to consider pre-exact categories that are not exact.

Example 4.4. There is another way to make a category of topological modules into a pre-exact category. Let $A$ be a $\widehat{\otimes}$-algebra, and let $\mathscr{C}$ be a full additive subcategory of $\mathrm{LCS}^{\wedge}$. We say that an exact pair $X \rightarrow Y \rightarrow Z$ in $A$ - $\bmod (\mathscr{C})$ is admissible if the sequence $0 \rightarrow X \rightarrow Y \rightarrow Z \rightarrow 0$ splits in $\mathscr{C}$. The resulting pre-exact category will be denoted by the same symbol $A$ - $\bmod (\mathscr{C})$. The pre-exact categories mod- $A(\mathscr{C})$ and $A$-mod- $A(\mathscr{C})$ are defined similarly. These are the basic categories of Topological Homology (see [20]). In fact, it is easy to show that the above categories are exact in the sense of Quillen.

Let $\mathscr{A}$ and $\mathscr{B}$ be pre-exact categories, and let $F: \mathscr{A} \rightarrow \mathscr{B}$ be an additive functor. We say that $F$ is exact, if for each admissible pair $X \rightarrow Y \rightarrow Z$ in $\mathscr{A}$ the pair $F X \rightarrow F Y \rightarrow F Z$ is admissible in $\mathscr{B}$. A standard argument shows that $F$ is exact if and only if for each admissible complex $C_{\bullet}$ in $\mathscr{A}$ the complex $F\left(C_{\bullet}\right)$ is admissible in $\mathscr{B}$.

Definition 4.5 (Helemskii $[\mathbf{1 7}, \mathbf{1 9}, \mathbf{2 0}]$ ). Let $A$ be a Fréchet algebra. A left Fréchet $A$-module $X$ is flat if the functor

$$
(\cdot) \underset{A}{\widehat{\otimes}} X: \bmod -A(\mathrm{Fr}) \rightarrow \mathrm{Vect}
$$

is exact. The module $X$ is strictly flat if the functor

$$
(\cdot) \widehat{\otimes}_{A} X: \underline{\bmod } A(\mathrm{Fr}) \rightarrow \text { Vect }
$$

is exact. A right Fréchet $A$-module (respectively, a Fréchet $A$-bimodule) is flat if it is flat as a left Fréchet module over $A^{\text {op }}$ (respectively, over $A^{e}$ ). Strictly flat right Fréchet modules and bimodules are defined similarly.

Remark 4.6. As easily follows from the Open Mapping Theorem, a pair $X \rightarrow Y \rightarrow Z$ in Fr is exact if and only if it is exact in Vect. Therefore we may consider the functors appearing in Definition 4.5 as functors with values in Fr. This will lead to an equivalent definition.

Remark 4.7. Suppose that $A$ is a unital Fréchet algebra and $X$ is a unital left Fréchet $A$-module. Then $X$ is flat (respectively, strictly flat) if and only if the restriction of (4) (respectively, of (5)) to unmod- $A$ (Fr) (respectively, to unmod- $A(\mathrm{Fr})$ ) is exact. Indeed, each right Fréchet $A$-module $Y$ can be decomposed as $Y=Y^{\text {un }} \oplus Y^{\text {ann }}$, where $Y^{\text {un }}$ is a unital $A$-module and $Y^{\text {ann }}$ is an annihilator $A$-module (i.e., $y \cdot a=0$ for all $\left.y \in Y^{\mathrm{ann}}, a \in A\right)$. Specifically,

$$
Y^{\mathrm{un}}=\{y \in Y: y \cdot 1=y\} \quad \text { and } \quad Y^{\mathrm{ann}}=\{y \in Y: y \cdot 1=0\} .
$$

It is easy to see that this decomposition is natural, so that each complex $Y_{\bullet}$ of right Fréchet $A$-modules decomposes as $Y_{\bullet}=Y_{\bullet}^{\text {un }} \oplus Y_{\bullet}^{\text {ann }}$. Clearly, $Y_{\bullet}$ is admissible in mod- $A(\mathrm{Fr})$ (respectively, in $\underline{\bmod } A(\mathrm{Fr}))$ if and only if both $Y_{\bullet}^{\text {un }}$ and $Y_{\bullet}^{\text {ann }}$ are. On 
the other hand, if $X$ is unital, then $Y_{\bullet}^{\text {ann }} \widehat{\otimes}_{A} X=0$, and so $Y_{\bullet} \widehat{\otimes}_{A} X \cong Y_{\bullet}^{\text {un }} \widehat{\otimes}_{A} X$. This proves the claim.

Remark 4.8. Certainly, Definition 4.5 makes sense for more general module categories of the form $A-\bmod (\mathscr{C})$ and $A-\bmod (\mathscr{C})$. However, for the reasons explained in Remark 7.5 below, we shall consider flatness and strict flatness only in categories of Fréchet modules over Fréchet algebras.

Proposition 4.9. Let $\varphi: A \rightarrow B$ be a Fréchet algebra homomorphism, and let $X$ be a flat (respectively, strictly flat) left Fréchet $A$-module. Then $B_{+} \widehat{\otimes}_{A} X$ is flat (respectively, strictly flat) in $B-\bmod (\mathrm{Fr})$.

Proof. The functors $(\cdot) \widehat{\otimes}_{B} B_{+} \widehat{\otimes}_{A} X$ and $(\cdot) \widehat{\otimes}_{A} X$ on mod- $B(\mathrm{Fr})$ are obviously isomorphic.

Proposition 4.10. Let $A$ be a Fréchet algebra, and let

$$
0 \rightarrow X \rightarrow Y \rightarrow Z \rightarrow 0
$$

be an admissible sequence in $A-\bmod (\mathrm{Fr})$. Suppose that $Z$ is flat. Then $X$ is flat if and only if $Y$ is flat.

Proof. This is a special case of [10, Proposition 3.1.15].

Flat modules are traditionally considered within the category of Banach modules over a Banach algebra. The definition of a flat Banach module, as given in $[\mathbf{1 7}, \mathbf{1 9}, \mathbf{2 0}]$, is formally different from Definition 4.5 , as the domain of the functor $(\cdot) \widehat{\otimes}_{A} X$ is taken to be $\bmod -A(\mathrm{Ban})$ rather than $\bmod -A(\mathrm{Fr})$. The following proposition shows that the two definitions of flatness are consistent.

Proposition 4.11. Let $A$ be a Banach algebra, and let $X$ be a left Banach A-module. Then the following conditions are equivalent:

(i) the functor

$$
(\cdot) \underset{A}{\widehat{\otimes}} X: \bmod -A(\mathrm{Fr}) \rightarrow \text { Vect }
$$

is exact (i.e., $X$ is flat in the sense of Definition 4.5);

(ii) the functor

$$
(\cdot) \underset{A}{\widehat{\otimes}} X: \bmod -A(\text { Ban }) \rightarrow \text { Vect }
$$

is exact (i.e., $X$ is flat in the sense of [17]).

Proof. Clearly, (i) implies (ii). Conversely, suppose that (ii) holds, and let $Y_{\bullet}$ be an admissible complex in mod- $A(\mathrm{Fr})$. In order to prove that $Y_{\bullet} \widehat{\otimes}_{A} X$ is exact, it suffices to prove that $\left(Y_{\bullet} \widehat{\otimes}_{A} X\right)^{*}$ is exact (see, e.g., $\left.[\mathbf{3 5}, 26.4]\right)$. By Proposition 3.2, we have an isomorphism

$$
\left(Y_{\bullet} \widehat{\otimes} X\right)^{*} \cong \mathbf{h}_{A}\left(Y_{\bullet}, X^{*}\right) .
$$

Since $X^{*}$ is injective in mod- $A(\operatorname{Ban})\left[\mathbf{2 0}\right.$, VII.1.14], it follows that $X^{*}$ is a retract of 
$\mathscr{L}\left(A_{+}, X^{*}\right)$ in $\bmod -A($ Ban $)[\mathbf{2 0}$, III.1.31]. On the other hand,

$$
\mathbf{h}_{A}\left(Y_{\bullet}, \mathscr{L}\left(A_{+}, X^{*}\right)\right) \cong \mathscr{L}\left(Y_{\bullet}, X^{*}\right)
$$

(see [55, Proposition 3.2]), and the latter complex is obviously exact. This completes the proof.

A similar statement holds for strictly flat Banach modules.

Proposition 4.12. Let $A$ be a Banach algebra, and let $X$ be a left Banach A-module. Then the following conditions are equivalent:

(i) the functor

$$
(\cdot) \widehat{\otimes}_{A} X: \underline{\bmod }-A(\mathrm{Fr}) \rightarrow \text { Vect }
$$

is exact (i.e., $X$ is strictly flat in the sense of Definition 4.5);

(ii) the functor

$$
(\cdot) \underset{A}{\widehat{\otimes}} X: \underline{\bmod }-A(\operatorname{Ban}) \rightarrow \operatorname{Vect}
$$

is exact (i.e., $X$ is strictly flat in the sense of [19]).

Proof. Suppose that (ii) holds, and let $Y_{\bullet}$ be an exact complex in mod $A(\mathrm{Fr})$. As in Proposition 4.11, we have to prove that $\mathbf{h}_{A}\left(Y_{\bullet}, X^{*}\right)$ is exact. By [20, VII.1.14], $X^{*}$ is strictly injective in mod- $A$ (Ban). Choose an injective Banach space $E$ of the form $E=\ell^{\infty}(S)$ and an isometric embedding $\varphi: X^{*} \rightarrow E$. Define an $A$-module morphism $\varkappa$ to be the composition

$$
X^{*} \stackrel{\nu}{\rightarrow} \mathscr{L}\left(A_{+}, X^{*}\right) \stackrel{\varphi_{*}}{\longrightarrow} \mathscr{L}\left(A_{+}, E\right),
$$

where $\nu$ is the canonical embedding of $X^{*}$ into $\mathscr{L}\left(A_{+}, X^{*}\right)$ given by

$$
\nu(f)(a)=f \cdot a\left(f \in X^{*}, a \in A_{+}\right)
$$

(see [20, Section III.1.4]), and $\varphi_{*}$ is induced by $\varphi$ in the obvious way. Since both $\nu$ and $\varphi_{*}$ are topologically injective, so is $\varkappa=\varphi_{*} \nu$. Since $X^{*}$ is strictly injective in mod- $A$ (Ban), there exists an $A$-module morphism $\sigma: \mathscr{L}\left(A_{+}, E\right) \rightarrow X^{*}$ such that $\sigma \varkappa=$ $1_{X^{*}}[\mathbf{2 0}, \mathrm{VII} .1 .13]$. Therefore $X^{*}$ is a retract of $\mathscr{L}\left(A_{+}, E\right)$ in $\bmod -A(\operatorname{Ban})$. On the other hand,

$$
\mathbf{h}_{A}\left(Y_{\bullet}, \mathscr{L}\left(A_{+}, E\right)\right) \cong \mathscr{L}\left(Y_{\bullet}, E\right)
$$

(see [55, Proposition 3.2]), and the latter complex is exact due to the injectivity of $E=\ell^{\infty}(S)$ in $\operatorname{Fr}$ (see, e.g., [38, Section 4] or [56, 2.2.1]). This completes the proof.

Recall that a Banach algebra $A$ is amenable [27] if for each Banach $A$-bimodule $X$ every continuous derivation from $A$ to the dual bimodule, $X^{*}$, is inner. As was shown by Helemskii and Sheinberg [18] (see also [20, VII.2.17]), $A$ is amenable if and only if $A_{+}$is a flat Banach $A$-bimodule.

By definition $[\mathbf{2 2}, \mathbf{2 3}]$, a Fréchet algebra $A$ is amenable (respectively, biflat) if $A_{+}$ (respectively, $A$ ) is a flat Fréchet $A$-bimodule. A number of interesting characterizations of biflat Banach algebras was given by Selivanov [52].

Using Proposition 4.11, we get the following. 
Corollary 4.13. If $A$ is an amenable (respectively, biflat) Banach algebra, then $A$ is amenable (respectively, biflat) when considered as a Fréchet algebra.

Proposition 4.14. Let $\varphi: A \rightarrow B$ be a Fréchet algebra homomorphism. Suppose that $A$ is amenable and that the map

$$
B_{+} \widehat{\otimes} B_{+} B_{+} \rightarrow B_{+}, \quad b_{1} \otimes b_{2} \mapsto b_{1} b_{2}
$$

is bijective. Then $B$ is amenable.

Proof. We have

$$
B^{e} \underset{A^{e}}{\widehat{\otimes}} A_{+} \cong B_{+} \underset{A_{+}}{\widehat{\otimes}} A_{+} \underset{A_{+}}{\widehat{\otimes}} B_{+} \cong B_{+} \underset{A_{+}}{\widehat{\otimes}} B_{+} \cong B_{+},
$$

so the result follows from Proposition 4.9.

Remark 4.15. It can be shown that (6) is bijective if and only if $\varphi$ is an epimorphism in the category of Fréchet algebras (cf. [54, XI.1.2]). Note that this condition is satisfied whenever $\varphi$ has dense range.

Given a Fréchet algebra $A$ and a Fréchet $A$-bimodule $X$, consider the chain complex

$$
0 \leftarrow C_{0}(A, X) \stackrel{d_{1}}{\longleftarrow} C_{1}(A, X) \stackrel{d_{2}}{\longleftarrow} \cdots \stackrel{d_{n}}{\longleftarrow} C_{n}(A, X) \stackrel{d_{n+1}}{\longleftarrow} \cdots,
$$

where $C_{0}(A, X)=X, C_{n}(A, X)=X \widehat{\otimes} \underbrace{A \widehat{\otimes} \cdots \widehat{\otimes} A}_{n}$ for $n \geqslant 1$, and the differentials are given by

$$
\begin{aligned}
d_{n}\left(x \otimes a_{1} \otimes \cdots \otimes a_{n}\right)= & x \cdot a_{1} \otimes a_{2} \otimes \cdots \otimes a_{n} \\
& +\sum_{k=1}^{n-1}(-1)^{k} x \otimes a_{1} \otimes \cdots \otimes a_{k} a_{k+1} \otimes \cdots \otimes a_{n} \\
& +(-1)^{n} a_{n} \cdot x \otimes a_{1} \otimes \cdots \otimes a_{n-1}, \\
d_{1}(x \otimes a)= & x \cdot a-a \cdot x .
\end{aligned}
$$

By definition [15] (see also $[\mathbf{2 0 , 6}]$ ), the $n$th homology of $(7)$ is denoted by $\mathscr{H}_{n}(A, X)$ and is called the nth Hochschild homology group of $A$ with coefficients in $X$.

By [20, VII.2.17], $A$ is amenable if and only if for each $X \in A$-mod- $A(\mathrm{Fr})$ the space $\mathscr{H}_{0}(A, X)$ is Hausdorff, and $\mathscr{H}_{n}(A, X)=0$ for all $n \geqslant 1$. Denote by $\overline{[A, X]} \subset X$ the closure of the image of $d_{1}$. Then it follows from the above that $A$ is amenable if and only if the augmented complex

$$
0 \leftarrow X / \overline{[A, X]} \leftarrow C_{\bullet}(A, X)
$$

is exact.

\section{Approximate identities and approximate diagonals in topo- logical algebras}

In this section we collect some general facts on approximate identities and approximate diagonals. Although these facts are well known in the Banach algebra case (see, e.g., $[6,39])$, we give full proofs in most cases for the sake of completeness. 
Recall that a net $\left(e_{\alpha}\right)_{\alpha \in \Lambda}$ in a topological algebra $A$ is a right approximate identity (a right a.i. for short) if $a=\lim _{\alpha} a e_{\alpha}$ for each $a \in A$. Left approximate identities are defined similarly. A net $\left(e_{\alpha}\right)_{\alpha \in \Lambda}$ is a two-sided approximate identity (or just an approximate identity) if it is both a left and a right approximate identity. An approximate identity $\left(e_{\alpha}\right)_{\alpha \in \Lambda}$ (right, left, or two-sided) is bounded if the set $\left\{e_{\alpha}\right\}_{\alpha \in \Lambda}$ is bounded. The expression "bounded approximate identity" is abbreviated as "b.a.i.", as usual.

We start with a simple observation. Let us recall that a Hausdorff locally convex space $E$ is semi-Montel if each bounded subset of $E$ is relatively compact.

Lemma 5.1. Let $A$ be a semi-Montel topological algebra with a right b.a.i. Then $A$ has a right identity.

Proof. Let $\left(e_{\alpha}\right)_{\alpha \in \Lambda}$ be a right b.a.i. in $A$. By assumption, the set $\overline{\left\{e_{\alpha}\right\}_{\alpha \in \Lambda}}$ is compact, so that there exists a subnet $\left(e_{\alpha^{\prime}}\right)_{\alpha^{\prime} \in \Lambda^{\prime}}$ of $\left(e_{\alpha}\right)$ convergent to $e \in A$. Then for each $a \in A$, the net $\left(a e_{\alpha^{\prime}}\right)$ converges to $a$ and to $a e$ simultaneously. Hence $a=a e$, and so $e$ is a right identity in $A$.

Proposition 5.2. Let $A$ be a topological algebra. Then:

(i) A has a right a.i. if and only if, for each finite subset $F \subset A$ and each 0neighborhood $U \subset A$, there exists $b \in A$ such that $a-a b \in U$ for all $a \in F$;

(ii) $A$ has a right b.a.i. if and only if there exists a bounded subset $B \subset A$ such that for each finite subset $F \subset A$ and each 0-neighborhood $U \subset A$ there exists $b \in B$ such that $a-a b \in U$ for all $a \in F$.

Proof. If $\left(e_{\alpha}\right)$ is a right a.i. (respectively, a right b.a.i.) in $A$, then $a-a e_{\alpha}$ converges to 0 uniformly on finite subsets of $A$. Therefore the element $b=e_{\alpha}$ satisfies (i) (respectively, (ii)) for $\alpha$ large enough. To prove the converse, consider the set $S$ of all pairs $(U, F)$, where $U \subset A$ is a 0 -neighborhood and $F \subset A$ is a finite set, and define an order on $S$ by

$$
\left(U_{1}, F_{1}\right) \leqslant\left(U_{2}, F_{2}\right) \Longleftrightarrow U_{1} \supset U_{2} \text { and } F_{1} \subset F_{2} \text {. }
$$

Thus $S$ becomes a directed set. For each $\alpha=(U, F) \in S$, find $e_{\alpha} \in A$ (respectively, $\left.e_{\alpha} \in B\right)$ such that $a-a e_{\alpha} \in U$ for all $a \in F$. Then it is clear that $\left(e_{\alpha}\right)$ is a right a.i. (respectively, a right b.a.i.) in $A$.

Remark 5.3. Proposition 5.2 has obvious "left" and "two-sided" versions. In particular, $A$ has a two-sided (bounded) a.i. if and only if (there exists a bounded subset $B \subset A$ such that) for each finite subset $F \subset A$ and each 0-neighborhood $U \subset A$ there exists $b \in A$ (respectively, $b \in B$ ) such that $a-a b \in U$ and $a-b a \in U$ for all $a \in F$.

Remark 5.4. As in the case of Banach algebras, it can be shown that if a hypotopological algebra has a left and a right b.a.i., then it has a two-sided b.a.i. We omit the proof, as we do not use this result in the sequel.

Proposition 5.5. Let $A$ be a left hypotopological algebra. The following conditions are equivalent:

(i) A has a right b.a.i.;

(ii) there exists a bounded set $B \subset A$ such that $a \in \overline{a B}$ for each $a \in A$. 
If, in addition, $A$ is locally convex, then (i) and (ii) are equivalent to

(iii) $A^{* *}$ has a right identity;

(iv) there exists $e \in A^{* *}$ such that $i_{A}(a) e=i_{A}($ a) for each $a \in A$.

Proof. (i) $\Longrightarrow$ (ii). This is clear.

(ii) $\Longrightarrow$ (i). Let $F=\left\{a_{1}, \ldots, a_{n}\right\} \subset A$ be a finite set and $U \subset A$ a 0-neighborhood. Choose a circled 0-neighborhood $V \subset A$ such that $V+V+V \subset U$ and a 0-neighbor-hood $W \subset V$ such that $W B \subset V$ and $F W \subset V$. For each $j=1, \ldots, n$, set $B_{j}=$ $\left(1_{+}-B\right)^{n-j} \subset A_{+}$. Since $A$ (and hence $A_{+}$) is left hypotopological, we see that $B_{j}$ is bounded (cf. Section 2 or $[\mathbf{2 6}]$ ). Let $W^{+} \subset A_{+}$be a 0-neighborhood such that $W^{+} \cap A=W$. For each $j=1, \ldots, n$, choose a 0 -neighborhood $W_{j}^{+} \subset A_{+}$such that $W_{j}^{+} B_{j} \subset W^{+}$. Setting $W_{j}=W_{j}^{+} \cap A$, we see that $W_{j} B_{j} \subset W$.

Using (ii), we can inductively find $u_{1}, \ldots, u_{n} \in B$ such that

$$
a_{j}\left(1_{+}-u_{1}\right) \cdots\left(1_{+}-u_{j}\right) \in W_{j} \quad(j=1, \ldots, n) .
$$

Define $v \in A$ by $1_{+}-v=\left(1_{+}-u_{1}\right) \cdots\left(1_{+}-u_{n}\right)$. Then

$$
\begin{aligned}
a_{j}\left(1_{+}-v\right)=a_{j}\left(1_{+}-u_{1}\right) \cdots\left(1_{+}-u_{j}\right)\left(1_{+}-u_{j+1}\right) & \cdots\left(1_{+}-u_{n}\right) \\
& \in W_{j} B_{j} \subset W \quad(j=1, \ldots, n) .
\end{aligned}
$$

Finally, choose $u \in B$ such that $v-v u \in W$. Then for each $j=1, \ldots, n$, we have

$$
\begin{aligned}
a_{j}-a_{j} u=\left(a_{j}-a_{j} v\right)+a_{j}(v-v u)-\left(a_{j}-a_{j} v\right) u & \\
& \in W+F W-W B \subset V+V+V \subset U .
\end{aligned}
$$

Applying Proposition 5.2, we see that $A$ has a right b.a.i.

Now suppose that $A$ is locally convex.

(i) $\Longrightarrow$ (iv). Let $\left(e_{\alpha}\right)_{\alpha \in \Lambda}$ be a right b.a.i. in $A$. Since $\left\{i_{A}\left(e_{\alpha}\right)\right\} \subset A^{* *}$ is equicontinuous, there exists a subnet $\left(e_{\alpha^{\prime}}\right)_{\alpha^{\prime} \in \Lambda^{\prime}}$ of $\left(e_{\alpha}\right)_{\alpha \in \Lambda}$ such that $\left(i_{A}\left(e_{\alpha^{\prime}}\right)\right)$ weak ${ }^{*}$ converges to $e \in A^{* *}$. This implies that $\left(i_{A}(a) i_{A}\left(e_{\alpha^{\prime}}\right)\right)$ weak ${ }^{*}$ converges to $i_{A}(a) e$ for each $a \in A\left([\mathbf{1 6}, 3.6]\right.$; see also Section 2). On the other hand, $a e_{\alpha^{\prime}} \rightarrow a$, so that $i_{A}(a) i_{A}\left(e_{\alpha^{\prime}}\right) \rightarrow i_{A}(a)$, and, finally, $i_{A}(a)=i_{A}(a) e$ for each $a \in A$.

(iv) $\Longrightarrow$ (iii). This follows form the weak* density of $i_{A}(A)$ in $A^{* *}$ and the weak* continuity of the first Arens product in the first variable ([16, 3.4]).

(iii) $\Longrightarrow$ (ii). Let $e \in A^{* *}$ be a right identity. By the bipolar theorem, there exists a convex bounded subset $B \subset A$ such that $e$ belongs to the weak* closure of $i_{A}(B)$. Using again $[\mathbf{1 6}, 3.6]$, we see that for each $a \in A$ the element $i_{A}(a) e=i_{A}(a)$ belongs to the weak ${ }^{*}$ closure of $i_{A}(a) i_{A}(B)=i_{A}(a B)$. Equivalently, $a$ belongs to the weak closure of $a B$. Since $a B$ is convex, this completes the proof of (ii).

Let $A$ be a $\widehat{\otimes}$-algebra, and let $\pi_{0}: A \widehat{\otimes} A \rightarrow A$ denote the product map. (We write $\pi_{0}$ instead of $\pi$ in order to make our notation consistent with that of [20, VII.2.2] and of Section 9.) Following [28], we say that a net $\left(M_{\alpha}\right)_{\alpha \in \Lambda}$ in $A \widehat{\otimes} A$ is an approximate diagonal for $A$ if $a \cdot M_{\alpha}-M_{\alpha} \cdot a \rightarrow 0$ and $\pi_{0}\left(M_{\alpha}\right) a \rightarrow a$ for each $a \in A$. An approximate diagonal $\left(M_{\alpha}\right)_{\alpha \in \Lambda}$ is bounded if the set $\left\{M_{\alpha}\right\}_{\alpha \in \Lambda}$ is bounded. An element $M \in(A \widehat{\otimes} A)^{* *}$ is a virtual diagonal for $A$ if $a \cdot M=M \cdot a$ and $\pi_{0}^{* *}(M) \cdot a=i_{A}(a)$ for each $a \in A$. (Here $(A \widehat{\otimes} A)^{* *}$ is considered as a semitopological $A$-bimodule; see Section 2.) 
In what follows, given $a \in A$ and $M \in A \widehat{\otimes} A$, we set $[a, M]=a \cdot M-M \cdot a$.

Remark 5.6. Our terminology slightly differs from that of [28]. Namely, our "bounded approximate diagonal" is just "approximate diagonal" in [28]. However, it has recently become clear that unbounded approximate diagonals are also of interest (cf. [7, 12]).

Lemma 5.7. Let $A$ be a $\widehat{\otimes}$-algebra. Then $A$ has a bounded approximate diagonal if and only if $A$ has a virtual diagonal.

We omit the proof, as it is similar to the Banach algebra case [28, 1.2] (see also [6, 2.9.64]).

Let us recall some standard notation (see, e.g., [51]). Suppose that $\mathscr{U}$ is a neighborhood base at 0 in $A$. Given $U \in \mathscr{U}$, let $\overline{\Gamma(U \otimes U)}$ denote the closure of the absolutely convex hull of the set

$$
U \otimes U=\{a \otimes b: a, b \in U\} \subset A \widehat{\otimes} A .
$$

Then $\{\overline{\Gamma(U \otimes U)}: U \in \mathscr{U}\}$ is a neighborhood base at 0 in $A \widehat{\otimes} A[\mathbf{5 1}]$.

Proposition 5.8. Let $A$ be a $\widehat{\otimes}$-algebra. Then:

(i) A has an approximate diagonal if and only if, for each finite subset $F \subset A$ and each 0-neighborhood $U \subset A$, there exists $M \in A \widehat{\otimes} A$ with $[a, M] \in \overline{\Gamma(U \otimes U)}$ and $\pi_{0}(M) a-a \in U$ for all $a \in F ;$

(ii) A has a bounded approximate diagonal if and only if there exists a bounded subset $B \subset A \widehat{\otimes} A$ such that for each finite subset $F \subset A$ and each 0 -neighborhood $U \subset A$ there exists $M \in B$ with $[a, M] \in \overline{\Gamma(U \otimes U)}$ and $\pi_{0}(M) a-a \in U$ for all $a \in F$.

The proof is similar to that of Proposition 5.2 and is therefore omitted.

\section{Locally bounded approximate identities and locally bound- ed approximate diagonals}

Definition 6.1. Let $A$ be a topological algebra. We say that:

(i) A has a right (respectively, left) locally bounded approximate identity if for each 0-neighborhood $U \subset A$ there exists $C>0$ such that for each finite subset $F \subset A$ there exists $b \in C U$ with $a-a b \in U$ (respectively, $a-b a \in U$ ) for all $a \in F$;

(ii) A has a two-sided locally bounded approximate identity (or just a locally bounded approximate identity) if for each 0-neighborhood $U \subset A$ there exists $C>0$ such that for each finite subset $F \subset A$ there exists $b \in C U$ with $a-a b \in U$ and $a-b a \in U$ for all $a \in F$.

We abbreviate "locally bounded approximate identity" as "locally b.a.i."

Remark 6.2. A slightly more convenient definition is as follows: $A$ has a right locally b.a.i. if for each 0-neighborhood $U \subset A$ there exists $C>0$ such that for each finite subset $F \subset A$ and each $\varepsilon>0$ there exists $b \in C U$ with $a-a b \in \varepsilon U$ for all $a \in F$. To show that these definitions are equivalent, it suffices to substitute $\varepsilon^{-1} F$ for $F$ in Definition 6.1. It is also clear that instead of considering arbitrary 0-neighborhoods in $A$, we can restrict to 0 -neighborhoods belonging to some family $\mathscr{U}$ such that $\{t U: t>0, U \in \mathscr{U}\}$ is a neighborhood base at 0 . 
Remark 6.3. Let $A$ be a locally convex algebra, and let $\left\{\|\cdot\|_{\lambda}: \lambda \in \Lambda\right\}$ be a directed family of seminorms generating the topology of $A$. Using Remark 6.2 , we see that $A$ has a right locally b.a.i. if and only if there exists a family $\left\{C_{\lambda}: \lambda \in \Lambda\right\}$ of positive reals such that for each finite subset $F \subset A$, each $\lambda \in \Lambda$, and each $\varepsilon>0$ there exists $b \in A$ with $\|b\|_{\lambda} \leqslant C_{\lambda}$ and $\|a-a b\|_{\lambda}<\varepsilon$ for all $a \in F$. Similar statements hold for left and for two-sided locally b.a.i.'s.

Let us remark that the above characterization of locally b.a.i.'s in terms of seminorms was taken as definition in [45]. We thank A. Mallios for suggesting us to give a definition applicable to arbitrary (not necessarily locally convex) topological algebras (see Definition 6.1).

Remark 6.4. Taking into account Proposition 5.2, we see that the existence of a right (respectively, left, two-sided) b.a.i. implies the existence of a right (respectively, left, two-sided) locally b.a.i., which, in turn, implies the existence of a right (respectively, left, two-sided) a.i.

Remark 6.5. If $A$ is normable, then the notions of "bounded" and "locally bounded" a.i.'s are obviously equivalent (cf. Remark 6.3).

Proposition 6.6. Let $\varphi: A \rightarrow B$ be a continuous homomorphism of topological algebras. Suppose that $A$ has a right locally b.a.i. Then each of the following conditions implies that $B$ has a right locally b.a.i.:

(i) $\varphi$ is onto;

(ii) $\varphi$ has dense range, and the multiplication in $B$ is jointly continuous.

Proof. We prove (ii), with the proof of (i) being similar. Given a 0-neighborhood $V \subset B$, find circled 0-neighborhoods $V_{1} \subset V$ and $V_{2} \subset V_{1}$ such that $V_{1}+V_{1}+V_{1} \subset V$ and $V_{2}^{2} \subset V_{1}$. Set $U=\varphi^{-1}\left(V_{2}\right)$, and let $C>0$ be as in Definition 6.1. Without loss of generality, we assume that $C \geqslant 1$. Given a finite set $F^{\prime} \subset B$, find a finite set $F \subset A$ such that $F^{\prime} \subset \varphi(F)+C^{-1} V_{2}$. Then there exists $b \in C U$ such that $a-a b \in U$ for each $a \in F$. Take any $a^{\prime} \in F^{\prime}$ and choose $a \in F$ satisfying $a^{\prime}-\varphi(a) \in C^{-1} V_{2}$. Then $b^{\prime}=\varphi(b) \in C V_{2} \subset C V$, and

$$
\begin{aligned}
a^{\prime}-a^{\prime} b^{\prime}=\varphi(a-a b)+\left(a^{\prime}-\varphi(a)\right) & +\left(\varphi(a)-a^{\prime}\right) b^{\prime} \\
& \in V_{1}+V_{1}+C^{-1} V_{2} \cdot C V_{2} \subset V_{1}+V_{1}+V_{1} \subset V .
\end{aligned}
$$

The rest is clear.

Proposition 6.7. Let $A$ be an Arens-Michael algebra. Then the following conditions are equivalent:

(i) A has a right locally b.a.i.;

(ii) for each Banach algebra $B$ such that there exists a continuous homomorphism $\varphi: A \rightarrow B$ with dense range, the algebra $B$ has a right b.a.i.

Proof. (i) $\Longrightarrow$ (ii). This follows from Proposition 6.6 and Remark 6.5.

(ii) $\Longrightarrow$ (i). Let $U \subset A$ be an absolutely convex, idempotent 0 -neighborhood. By assumption, the Banach algebra $A_{U}$ has a right b.a.i. Hence the same is true of the dense subalgebra $A_{U}^{0}=\operatorname{Im} \tau_{U} \subset A_{U}$ (see $\left.[\mathbf{8}, 1.4]\right)$. Therefore there exists $C>0$ such 
that for each finite set $F \subset A$ there exists $b \in A$ such that $\left\|\tau_{U}(b)\right\|<C$ and that $\left\|\tau_{U}(a)-\tau_{U}(a) \tau_{U}(b)\right\|<1$ for each $a \in F$. This means exactly that $b \in C U$ and that $a-a b \in U$ for each $a \in F$. In view of Remark 6.2, this completes the proof.

Corollary 6.8. Let $A$ be a Arens-Michael algebra, and let $A=\lim A_{\lambda}$ be an ArensMichael decomposition of $A$. Then $A$ has a right locally b.a.i. if $\overleftarrow{\text { and }}$ only if each $A_{\lambda}$ has a right b.a.i.

Proof. Each continuous homomorphism $\varphi$ from $A$ to a Banach algebra $B$ uniquely factors through some $A_{\lambda}$. Therefore if $\varphi$ has dense range and $A_{\lambda}$ has a right b.a.i., then the same is true of $B$ (cf. $[\mathbf{8}, 1.4])$.

Remark 6.9. It is easy to see that Propositions 6.6 and 6.7 and Corollary 6.8 have obvious "right" and "two-sided" versions.

Remark 6.10. As in the case of Banach algebras, it can be shown that if a topological algebra with jointly continuous multiplication has a left and a right locally b.a.i., then it has a two-sided locally b.a.i. (cf. also Remark 5.4). We omit the proof, as we do not use this result in the sequel.

Definition 6.11. Let $A$ be a $\widehat{\otimes}$-algebra. We say that $A$ has a locally bounded approximate diagonal if for each 0-neighborhood $U \subset A$ there exists $C>0$ such that for each finite subset $F \subset A$ there exists $M \in C \overline{\Gamma(U \otimes U)}$ with $[a, M] \in \overline{\Gamma(U \otimes U)}$ and $\pi_{0}(M) a-a \in U$ for all $a \in F$.

Remark 6.12. Taking into account Proposition 5.8, we see that the existence of a bounded approximate diagonal implies the existence of a locally bounded approximate diagonal, which, in turn, implies the existence of an approximate diagonal.

Remark 6.13. For a Banach algebra, the notions of "bounded" and "locally bounded" approximate diagonals are equivalent by Proposition 5.8(ii).

Proposition 6.14. Let $\varphi: A \rightarrow B$ be a continuous homomorphism of $\widehat{\otimes}$-algebras. Suppose that $A$ has a locally bounded approximate diagonal and that $\varphi$ has dense range. Then $B$ has a locally bounded approximate diagonal.

Proof. Given an absolutely convex 0-neighborhood $V \subset B$, find an absolutely convex 0-neighborhood $V_{1} \subset B$ such that $V_{1} \cap V_{1}^{2} \cap \overline{V_{1}^{3}} \subset V / 3$. Set $U=\varphi^{-1}\left(V_{1}\right)$, and let $C>0$ be as in Definition 6.11. Without loss of generality, we assume that $C \geqslant 1$. Given a finite set $F^{\prime} \subset B$, find a finite set $F \subset A$ such that $F^{\prime} \subset \varphi(F)+C^{-1} V_{1}$. Then there exists $M \in C \overline{\Gamma(U \otimes U)}$ with $[a, M] \in \overline{\Gamma(U \otimes U)}$ and $\pi_{0}(M) a-a \in U$ for all $a \in F$.

Set $M^{\prime}=(\varphi \otimes \varphi)(M)$. Clearly, $M^{\prime} \in C \overline{\Gamma\left(V_{1} \otimes V_{1}\right)} \subset C \overline{\Gamma(V \otimes V)}$. Now take any $a^{\prime} \in F^{\prime}$ and choose $a \in F$ satisfying $a^{\prime}-\varphi(a) \in C^{-1} V_{1}$. We have

$$
\begin{aligned}
{\left[a^{\prime}, M^{\prime}\right] } & =(\varphi \otimes \varphi)[a, M]+\left[a^{\prime}-\varphi(a), M^{\prime}\right] \\
& \in \overline{\Gamma\left(V_{1} \otimes V_{1}\right)}+C^{-1} V_{1} \cdot C \overline{\Gamma\left(V_{1} \otimes V_{1}\right)}+C \overline{\Gamma\left(V_{1} \otimes V_{1}\right)} \cdot C^{-1} V_{1} \subset \overline{\Gamma(V \otimes V)} .
\end{aligned}
$$

Furthermore,

$$
\begin{aligned}
\pi_{0}\left(M^{\prime}\right) a^{\prime}-a^{\prime}=\varphi\left(\pi_{0}(M) a-a\right)+\left(\varphi(a)-a^{\prime}\right)+\pi_{0}\left(M^{\prime}\right)\left(a^{\prime}-\varphi(a)\right) \\
\in V_{1}+V_{1}+C \overline{V_{1}^{2}} C^{-1} V_{1} \subset V .
\end{aligned}
$$

The rest is clear. 
Proposition 6.15. Let $A$ be an Arens-Michael algebra. Then the following conditions are equivalent:

(i) A has a locally bounded approximate diagonal;

(ii) for each Banach algebra $B$ such that there exists a continuous homomorphism $\varphi: A \rightarrow B$ with dense range, the algebra $B$ has a bounded approximate diagonal.

Proof. (i) $\Longrightarrow$ (ii). This follows from Proposition 6.14 and Remark 6.13.

(ii) $\Longrightarrow$ (i). Let $U \subset A$ be an absolutely convex, idempotent 0-neighborhood. By assumption, the Banach algebra $A_{U}$ has a bounded approximate diagonal. Therefore there exists $C>0$ such that for each finite set $F \subset A$ there exists $M^{\prime} \in A_{U} \widehat{\otimes} A_{U}$ with

$$
\begin{gathered}
\left\|M^{\prime}\right\|<C, \\
\left\|\left[\tau_{U}(a), M^{\prime}\right]\right\|<1 \quad \forall a \in F, \\
\left\|\pi_{0}\left(M^{\prime}\right) \tau_{U}(a)-\tau_{U}(a)\right\|<1 \quad \forall a \in F .
\end{gathered}
$$

Clearly, the set of all $M^{\prime} \in A_{U} \widehat{\otimes} A_{U}$ satisfying (9)-(11) is open. On the other hand, $\left(\tau_{U} \otimes \tau_{U}\right)(A)$ is dense in $A_{U} \widehat{\otimes} A_{U}$, and so there exists $M \in A \widehat{\otimes} A$ such that $M^{\prime}=$ $\left(\tau_{U} \otimes \tau_{U}\right)(M)$ satisfies (9)-(11). This implies that $M$ satisfies the conditions of Definition 6.11 .

Corollary 6.16. Let $A$ be a Arens-Michael algebra, and let $A=\lim A_{\lambda}$ be an ArensMichael decomposition of $A$. Then $A$ has a locally bounded approximate diagonal if and only if each $A_{\lambda}$ has a bounded approximate diagonal.

\section{Strictly flat cyclic modules}

In this section we extend the first part of Helemskii and Sheinberg's Theorem 1.1 (specifically, the equivalence of conditions (i) and (ii)) to Arens-Michael algebras.

Lemma 7.1. Let $A$ be an Arens-Michael algebra, $X \in \bmod -A$, and $X=\varliminf_{\lim }\left(X_{\lambda}, \tau_{\lambda}^{\mu}\right)$ be an Arens-Michael decomposition of $X$. Suppose that $I \subset A$ is a closed left ideal. For each $\lambda$, consider the map

$$
\hat{\tau}_{\lambda}: X / \overline{X \cdot I} \rightarrow X_{\lambda} / \overline{X_{\lambda} \cdot I}, \quad x+\overline{X \cdot I} \mapsto \tau_{\lambda}(x)+\overline{X_{\lambda} \cdot I} .
$$

Then $\left(X_{\lambda} / \overline{X_{\lambda} \cdot I}, \hat{\tau}_{\lambda}\right)$ is the completion of $X / \overline{X \cdot I}$ with respect to the quotient seminorm of $\|\cdot\|_{\lambda}$. As a consequence, we have a topological isomorphism

$$
(X / \overline{X \cdot I})^{\sim} \cong \varliminf_{\varlimsup}\left(X_{\lambda} / \overline{X_{\lambda} \cdot I}, \hat{\tau}_{\lambda}^{\mu}\right)
$$

where the structure maps $\hat{\tau}_{\lambda}^{\mu}(\lambda \prec \mu)$ are defined by

$$
\hat{\tau}_{\lambda}^{\mu}: X_{\mu} / \overline{X_{\mu} \cdot I} \rightarrow X_{\lambda} / \overline{X_{\lambda} \cdot I}, \quad x+\overline{X_{\mu} \cdot I} \mapsto \tau_{\lambda}^{\mu}(x)+\overline{X_{\lambda} \cdot I} .
$$

Proof. Since $\tau_{\lambda}: X \rightarrow X_{\lambda}$ has dense range, the same is true of $\hat{\tau}_{\lambda}$. Therefore we need only prove that $\hat{\tau}_{\lambda}$ is isometric with respect to the quotient seminorm of $\|\cdot\|_{\lambda}$ on $X / \overline{X \cdot I}$ and the quotient norm on $X_{\lambda} / \overline{X_{\lambda} \cdot I}$. Denote the former seminorm by $\|\cdot\|_{\lambda}^{\prime}$ 
and the latter norm by $\|\cdot\|^{\prime}$. Then for each $x \in X$, we have

$$
\begin{aligned}
\|x+\overline{X \cdot I}\|_{\lambda}^{\prime} & =\inf \left\{\|x+y\|_{\lambda}: y \in \overline{X \cdot I}\right\}=\inf \left\{\left\|\tau_{\lambda}(x)+\tau_{\lambda}(y)\right\|: y \in \overline{X \cdot I}\right\} \\
& =\inf \left\{\left\|\tau_{\lambda}(x)+z\right\|: z \in \overline{X_{\lambda} \cdot I}\right\}=\left\|\tau_{\lambda}(x)+\overline{X_{\lambda} \cdot I}\right\|^{\prime} \\
& =\left\|\hat{\tau}_{\lambda}(x+\overline{X \cdot I})\right\|^{\prime},
\end{aligned}
$$

which proves the claim.

Theorem 7.2. Let $A$ be an Arens-Michael algebra and $I \subset A_{+}$a closed left ideal. Set $X=\left(A_{+} / I\right)^{\sim}$. Then the following conditions are equivalent:

(i) I has a right locally b.a.i.;

(ii) the functor $(\cdot) \widehat{\otimes}_{A} X: \underline{\bmod }-A \rightarrow \mathrm{LCS}^{\wedge}$ is exact;

(iii) the functor $(\cdot) \widehat{\otimes}_{A} X: \underline{\bmod }-A(\operatorname{Ban}) \rightarrow$ Ban is exact.

Proof. (i) $\Longrightarrow$ (ii). In view of Proposition 3.3 and Remark 4.1, it suffices to prove that for each $Y \in \underline{\bmod } A$ and each closed submodule $Z \subset Y$ the map

$$
Z \underset{A}{\widehat{\otimes}} X \rightarrow Y \underset{A}{\widehat{\otimes}} X
$$

is topologically injective. Choose an Arens-Michael decomposition $Y=\lim _{Y_{\lambda}}$ of $Y$, and, for each $\lambda$, set $Z_{\lambda}=\overline{\tau_{\lambda}(Z)}$, where $\tau_{\lambda}: Y \rightarrow Y_{\lambda}$ is the canonical map. Then it is easy to see that $Z=\lim Z_{\lambda}$ is an Arens-Michael decomposition of $Z$ (cf. also [9, 2.5.6]). Since each seminorm $\|\cdot\|_{\lambda}$ on $Y$ is $m$-compatible (see Section 2), there exists a continuous submultiplicative seminorm $\|\cdot\|_{\lambda}^{\circ}$ on $A$ such that $\|y \cdot a\|_{\lambda} \leqslant\|y\|_{\lambda}\|a\|_{\lambda}^{\circ}$ for each $y \in Y, a \in A$. Let $A_{\lambda}$ be the completion of $A$ with respect to $\|\cdot\|_{\lambda}^{\circ}$. Then it is clear that $Y_{\lambda}$ is a right Banach $A_{\lambda}$-module in a canonical way, and that $Z_{\lambda}$ is a closed $A_{\lambda}$-submodule of $X_{\lambda}$.

Let $\sigma_{\lambda}: A \rightarrow A_{\lambda}$ be the canonical map, and let $I_{\lambda}=\overline{\left(\sigma_{\lambda}\right)_{+}(I)}$. Clearly, $I_{\lambda}$ is a closed left ideal in $\left(A_{\lambda}\right)_{+}$. By Proposition 6.7, $I_{\lambda}$ has a right b.a.i., and so $X_{\lambda}=\left(A_{\lambda}\right)_{+} / I_{\lambda}$ is a strictly flat Banach $A_{\lambda}$-module (see Theorem 1.1). Therefore the map

$$
Z_{\lambda} \underset{A_{\lambda}}{\widehat{\otimes}} X_{\lambda} \rightarrow Y_{\lambda} \underset{A_{\lambda}}{\widehat{\otimes}} X_{\lambda}
$$

is topologically injective. Using the identifications

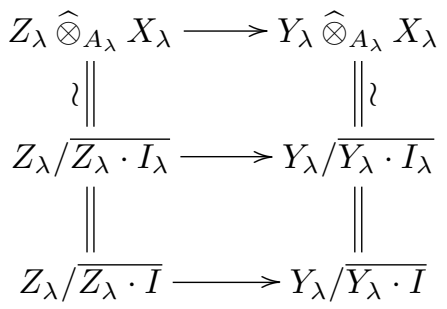

and taking the inverse limit, we get a topologically injective map

$$
\lim _{\lambda} Z_{\lambda} \overline{Z_{\lambda} \cdot I} \rightarrow \underset{\lim }{\longleftarrow} Y_{\lambda} / \overline{Y_{\lambda} \cdot I} \text {. }
$$

On the other hand, Lemma 7.1 and Proposition 3.1 imply that the latter map is 
identifiable with (12) as follows:

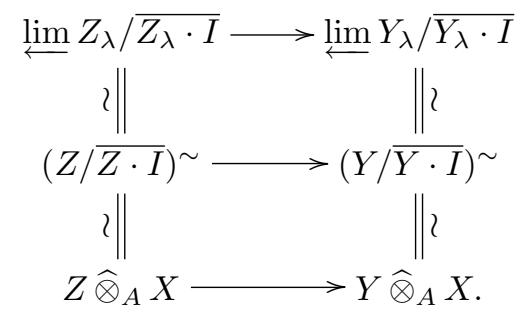

This proves (ii).

(ii) $\Longrightarrow$ (iii). This is clear.

(iii) $\Longrightarrow$ (i). Let $\varphi: A \rightarrow B$ be a homomorphism of $A$ to a Banach algebra $B$. Using the same argument as in Proposition 4.9, we see that the left Banach $B$-module $B_{+} \widehat{\otimes}_{A} X \cong B_{+} / \overline{B_{+} \cdot I}$ is strictly flat. Therefore the ideal $\overline{B_{+} \cdot I}$ of $B_{+}$has a right b.a.i.

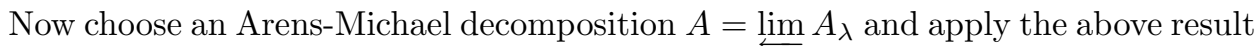
to the canonical homomorphism $\sigma_{\lambda}: A \rightarrow A_{\lambda}$. In this case, the ideal $I_{\lambda}=\overline{\left(A_{\lambda}\right)_{+} \cdot I}$ of $\left(A_{\lambda}\right)_{+}$is equal to the closure of $\left(\sigma_{\lambda}\right)_{+}(I)$ in $\left(A_{\lambda}\right)_{+}$, so that $I=\lim I_{\lambda}$ is an ArensMichael decomposition of $I$. By Corollary 6.8, $I$ has a right locally b.a.i., as required.

Corollary 7.3. Let $A$ be a Fréchet-Arens-Michael algebra and $I \subset A_{+}$a closed left ideal. Set $X=A_{+} / I$. Then the following conditions are equivalent:

(i) I has a right locally b.a.i.;

(ii) $X$ is a strictly flat Fréchet $A$-module.

Remark 7.4. Theorem 7.2 and Corollary 7.3 have obvious "unital" versions. Specifically, if $A$ is unital, then we can replace $A_{+}$by $A$ and "mod" by "unmod" everywhere in the statements of Theorem 7.2 and Corollary 7.3 (cf. also Remark 4.7).

Remark 7.5. In view of Theorem 7.2 and Corollary 7.3, it is tempting to call a left $\widehat{\otimes}$-module $X$ over a $\widehat{\otimes}$-algebra $A$ strictly flat if it satisfies condition (ii) of Theorem 7.2. However, it is not clear whether such a "strictly flat" module is always flat (which, by definition [20], means that the functor $(\cdot) \widehat{\otimes}_{A} X: \bmod -A \rightarrow$ Vect is exact). A possible way out of this situation is to redefine the notion of flatness as follows: $X \in A$ - $\bmod (\mathscr{C})$ is flat if the functor $(\cdot) \widehat{\otimes}_{A} X: \bmod -A(\mathscr{C}) \rightarrow \mathscr{C}$ is exact. If $\mathscr{C}=\mathrm{Fr}$ or $\mathscr{C}=$ Ban, this is equivalent to Definition 4.5 (cf. Remark 4.6). However, the disadvantage of the new definition is that it might be impossible to characterize such "flatness" in terms of the derived functors $\operatorname{Tor}_{n}$ (cf. [20]). That is why we do not consider flat and strictly flat $\widehat{\otimes}$-modules in the general setting, restricting ourselves to the metrizable case.

\section{Quasinormable Fréchet algebras}

Recall that a Hausdorff l.c.s. $E$ is quasinormable [14] if for each 0-neighborhood $U \subset E$ there exists a 0-neighborhood $V \subset U$ such that for each $\varepsilon>0$ there exists a 
bounded set $B \subset E$ satisfying $V \subset B+\varepsilon U$. In this section we prove that for quasinormable Fréchet algebras the notions of bounded and locally bounded approximate identities are equivalent.

Lemma 8.1. Let $\varphi: A \rightarrow B$ be a Banach algebra homomorphism with dense range. Then:

(i) $\varphi^{* *}\left(\operatorname{rann}\left(A^{* *}\right)\right) \subset \operatorname{rann}\left(B^{* *}\right)$;

(ii) if $e$ is a right identity in $A^{* *}$, then $\varphi^{* *}(e)$ is a right identity in $B^{* *}$.

Proof. If $a \in \operatorname{rann}\left(A^{* *}\right)$, then $b \varphi^{* *}(a)=0$ for all $b \in \operatorname{Im} \varphi^{* *}$. Since $\operatorname{Im} \varphi$ is dense in $B$, it follows that $\operatorname{Im} \varphi^{* *}$ is weak ${ }^{*}$ dense in $B^{* *}$. As the product in $B^{* *}$ is weak ${ }^{*}$ continuous with respect to the first variable, this implies that $b \varphi^{* *}(a)=0$ for all $b \in B^{* *}$, i.e., that $\varphi^{* *}(a) \in \operatorname{rann}\left(B^{* *}\right)$. Similarly, if $e$ is a right identity in $A^{* *}$, then $\varphi^{* *}(e)$ is a right identity for $\operatorname{Im} \varphi^{* *}$, and, by weak* continuity, for $B^{* *}$.

From now on, let $A$ be a Fréchet-Arens-Michael algebra, and $A=\lim \left(A_{n}, \tau_{n}^{m}\right)_{n \in \mathbb{N}}$ be an Arens-Michael decomposition of $A$. By Lemma 8.1, we have a well-defined inverse system $\left(\operatorname{rann}\left(A_{n}^{* *}\right), \sigma_{n}^{m}\right)$, where $\sigma_{n}^{m}$ is the restriction of $\left(\tau_{n}^{m}\right)^{* *} \operatorname{to} \operatorname{rann}\left(A_{m}^{* *}\right)$.

Lemma 8.2. Suppose that $A$ is quasinormable and has a right locally b.a.i. Then $\lim ^{1} \operatorname{rann}\left(A_{n}^{* *}\right)=0$.

Proof. Since $A$ is quasinormable, the direct system $\left(A_{n}^{*},\left(\tau_{n}^{m}\right)^{*}\right)$ is acyclic $[\mathbf{3 8}$, Proposition 7.5], and so $\lim ^{1} A_{n}^{* *}=0$ [38, Proposition 6.2]. By Palamodov's Theorem 2.1, for each $n \in \mathbb{N}$ there exists $m \geqslant n$ such that $\left(\tau_{n}^{k}\right)^{* *}\left(A_{k}^{* *}\right)$ is dense in $\left(\tau_{n}^{m}\right)^{* *}\left(A_{m}^{* *}\right)$ for all $k \geqslant m$.

For each $n \in \mathbb{N}$, set $R_{n}=\operatorname{rann}\left(A_{n}^{* *}\right)$. We claim that $\left(R_{n}, \sigma_{n}^{m}\right)$ satisfies the conditions of Palamodov's Theorem with the same $m=m(n)$. To prove the claim, fix $n \in \mathbb{N}$, and take any $k \geqslant m$. Since $A$ has a right locally b.a.i., Corollary 6.8 implies that $A_{k}$ has a right b.a.i., and so $A_{k}^{* *}$ has a right identity, say $e_{k}$. By Lemma $8.1, e_{m}=\left(\tau_{m}^{k}\right)^{* *}\left(e_{k}\right)$ and $e_{n}=\left(\tau_{n}^{m}\right)^{* *}\left(e_{m}\right)$ are right identities in $A_{m}^{* *}$ and $A_{n}^{* *}$, respectively.

Define $p_{k}: A_{k}^{* *} \rightarrow R_{k}$ by $p_{k}(a)=a-e_{k} a$. Clearly, $p_{k}$ is a projection onto $R_{k}$. Similarly, define projections

$$
p_{m}: A_{m}^{* *} \rightarrow R_{m}, a \mapsto a-e_{m} a, \quad \text { and } \quad p_{n}: A_{n}^{* *} \rightarrow R_{n}, a \mapsto a-e_{n} a .
$$

We obtain a commutative diagram

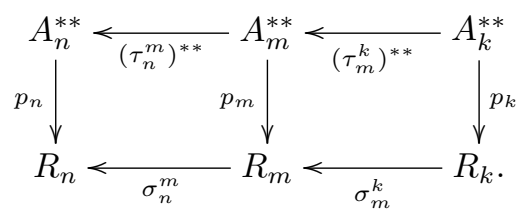

Therefore,

$$
\begin{aligned}
\overline{\sigma_{n}^{m}\left(R_{m}\right)}=\overline{\sigma_{n}^{m}\left(p_{m}\left(A_{m}^{* *}\right)\right)} & =\overline{p_{n}\left(\overline{\left(\tau_{n}^{m}\right)^{* *}\left(A_{m}^{* *}\right)}\right)} \\
& =\overline{p_{n}\left(\overline{\left(\tau_{n}^{k}\right)^{* *}\left(A_{k}^{* *}\right)}\right)}=\overline{\sigma_{n}^{k}\left(p_{k}\left(A_{k}^{* *}\right)\right)}=\overline{\sigma_{n}^{k}\left(R_{k}\right)} .
\end{aligned}
$$

Thus $\left(\operatorname{rann}\left(A_{n}^{* *}\right), \sigma_{n}^{m}\right)$ satisfies the conditions of Palamodov's Theorem 2.1, and so $\lim ^{1} \operatorname{rann}\left(A_{n}^{* *}\right)=0$. 
Remark 8.3. We note that Lemma 8.2 holds under a weaker assumption than the quasinormability of $A$. Specifically, it suffices to require that the direct system $\left(A_{n}^{*},\left(\tau_{n}^{m}\right)^{*}\right)$ be weakly acyclic [38]; see also [56, Chapter 6].

Theorem 8.4. Let $A$ be a quasinormable Fréchet-Arens-Michael algebra with a right locally b.a.i. Then A has a right b.a.i.

Proof. Let $A=\lim _{(}\left(A_{n}, \tau_{n}^{m}\right)_{n \in \mathbb{N}}$ be an Arens-Michael decomposition of $A$. For each $n \in \mathbb{N}$, consider the admissible sequences

$$
\begin{aligned}
& 0 \leftarrow \mathbb{C}_{\ell} \stackrel{\varepsilon_{A_{n}}}{\longleftarrow}\left(A_{n}\right)_{+} \leftarrow A_{n} \leftarrow 0, \\
& 0 \rightarrow \mathbb{C}_{r} \stackrel{\varepsilon_{A_{n}}^{*}}{\longrightarrow}\left(A_{n}\right)_{+}^{*} \rightarrow A_{n}^{*} \rightarrow 0
\end{aligned}
$$

in $A_{n}$ - $\bmod (\operatorname{Ban})$ and in $\bmod -A_{n}(\operatorname{Ban})$, respectively. Since $A$ has a right locally b.a.i., Corollary 6.8 implies that $A_{n}$ has a right b.a.i., and so $\mathbb{C}_{r}$ is injective in mod- $A_{n}$ (Ban) [20, VII.1.20]. Hence the sequence

$$
0 \rightarrow \mathbf{h}_{A_{n}}\left(A_{n}^{*}, \mathbb{C}_{r}\right) \rightarrow \mathbf{h}_{A_{n}}\left(\left(A_{n}\right)_{+}^{*}, \mathbb{C}_{r}\right) \rightarrow \mathbf{h}_{A_{n}}\left(\mathbb{C}_{r}, \mathbb{C}_{r}\right) \rightarrow 0
$$

is exact. Since $\tau_{n}: A \rightarrow A_{n}$ has dense range, we have $\mathbf{h}_{A_{n}}=\mathbf{h}_{A}$ in the above sequence. Using the isomorphism $\mathbf{h}_{A_{n}}\left(\mathbb{C}_{r}, \mathbb{C}_{r}\right) \cong \mathbb{C}$, we may identify (13) with the sequence

$$
0 \rightarrow \mathbf{h}_{A}\left(A_{n}^{*}, \mathbb{C}_{r}\right) \rightarrow \mathbf{h}_{A}\left(\left(A_{n}\right)_{+}^{*}, \mathbb{C}_{r}\right) \stackrel{\Phi_{n}}{\longrightarrow} \mathbb{C} \rightarrow 0
$$

where $\Phi_{n}(f)=f\left(\varepsilon_{A_{n}}\right)$ for each $f \in \mathbf{h}_{A}\left(\left(A_{n}\right)_{+}^{*}, \mathbb{C}_{r}\right)$.

Using (1), we see that $\mathbf{h}_{A}\left(A_{n}^{*}, \mathbb{C}_{r}\right)=\mathbf{h}_{A_{n}}\left(A_{n}^{*}, \mathbb{C}_{r}\right)=\operatorname{rann}\left(A_{n}^{* *}\right)$, and it is clear that for each $m \geqslant n$ the map

$$
\mathbf{h}_{A}\left(A_{m}^{*}, \mathbb{C}_{r}\right) \rightarrow \mathbf{h}_{A}\left(A_{n}^{*}, \mathbb{C}_{r}\right), \quad f \mapsto f \circ\left(\tau_{n}^{m}\right)^{*}
$$

coincides with the map $\sigma_{n}^{m}$ defined before Lemma 8.2. Therefore taking the inverse limit in (14) and applying Lemma 8.2 we get an exact sequence

$$
0 \rightarrow \lim _{\longleftarrow} \mathbf{h}_{A}\left(A_{n}^{*}, \mathbb{C}_{r}\right) \rightarrow \lim _{\longleftarrow} \mathbf{h}_{A}\left(\left(A_{n}\right)_{+}^{*}, \mathbb{C}_{r}\right) \rightarrow \mathbb{C} \rightarrow 0 .
$$

Since $A$ is quasinormable (hence distinguished [35, 26.19]), the canonical linear map $\lim _{n} A_{n}^{*} \rightarrow A^{*}$ is an isomorphism of locally convex spaces [35, 25.13]. Clearly, this is also $\overrightarrow{\text { an }}$ isomorphism in the category of right locally convex $A$-modules. Hence we obtain vector space isomorphisms

$$
\lim _{\mathbf{h}_{A}}\left(A_{n}^{*}, \mathbb{C}_{r}\right) \cong \mathbf{h}_{A}\left(\lim _{\longrightarrow} A_{n}^{*}, \mathbb{C}_{r}\right) \cong \mathbf{h}_{A}\left(A^{*}, \mathbb{C}_{r}\right) .
$$

Similarly, $\lim _{\longleftarrow} \mathbf{h}_{A}\left(\left(A_{n}\right)_{+}^{*}, \mathbb{C}_{r}\right) \cong \mathbf{h}_{A}\left(A_{+}^{*}, \mathbb{C}_{r}\right)$ as vector spaces. Therefore (15) can be identified with

$$
0 \rightarrow \mathbf{h}_{A}\left(A^{*}, \mathbb{C}_{r}\right) \rightarrow \mathbf{h}_{A}\left(A_{+}^{*}, \mathbb{C}_{r}\right) \stackrel{\Phi}{\rightarrow} \mathbb{C} \rightarrow 0,
$$

where $\Phi(f)=f\left(\varepsilon_{A}\right)$ for each $f \in \mathbf{h}_{A}\left(A_{+}^{*}, \mathbb{C}_{r}\right)$. 
As (16) is exact, there exists $f \in \mathbf{h}_{A}\left(A_{+}^{*}, \mathbb{C}_{r}\right)$ such that $f\left(\varepsilon_{A}\right)=1$. This is equivalent to say that the sequence

$$
0 \rightarrow \mathbb{C}_{r} \stackrel{\varepsilon_{A}^{*}}{\longrightarrow} A_{+}^{*} \stackrel{j_{A}^{*}}{\longrightarrow} A^{*} \rightarrow 0
$$

splits in the category of right locally convex $A$-modules. Therefore the dual sequence,

$$
0 \leftarrow \mathbb{C}_{\ell} \stackrel{\varepsilon_{A}^{* *}}{\longleftarrow} A_{+}^{* *} \stackrel{j_{A}^{* *}}{\longleftarrow} A^{* *} \leftarrow 0,
$$

splits in $A$-mod(Fr); thus there exists a left Fréchet $A$-module morphism $\sigma: A_{+}^{* *} \rightarrow A^{* *}$ such that $\sigma j_{A}^{* *}=1_{A^{* *}}$. Set $e=\sigma\left(i_{A_{+}}\left(1_{+}\right)\right) \in A^{* *}$. For each $a \in A$, we have

$$
i_{A}(a) e=a \cdot e=\sigma\left(i_{A_{+}}\left(a \cdot 1_{+}\right)\right)=\sigma\left(i_{A_{+}}\left(j_{A}(a)\right)=\sigma\left(j_{A}^{* *}\left(i_{A}(a)\right)=i_{A}(a) .\right.\right.
$$

By Proposition 5.5, this is equivalent to say that $A$ has a right b.a.i., as required.

\section{Flat Fréchet modules and amenable Fréchet algebras}

Part (iii) of Helemskii and Sheinberg's Theorem 1.1 can be generalized to ArensMichael algebras as follows.

Theorem 9.1. Let $A$ be an Arens-Michael algebra and $I \subset A_{+}$a closed left ideal. Set $X=\left(A_{+} / I\right)^{\sim}$. Suppose that there exists an Arens-Michael decomposition $A=$ $\varliminf_{\varlimsup}\left(A_{\lambda}, \sigma_{\mu}^{\lambda}\right)_{\lambda \in \Lambda}$ such that for each $\lambda \in \Lambda$ the ideal $I_{\lambda}=\overline{\left(\sigma_{\lambda}\right)_{+}(I)}$ is weakly complemented in $\left(A_{\lambda}\right)_{+}$. Then the following conditions are equivalent:

(i) I has a right locally b.a.i.;

(ii) the functor $(\cdot) \widehat{\otimes}_{A} X: \underline{\bmod } A \rightarrow \mathrm{LCS}^{\wedge}$ is exact;

(iii) the functor $(\cdot) \widehat{\otimes}_{A} X: \underline{\bmod } A(\operatorname{Ban}) \rightarrow$ Ban is exact;

(iv) the functor $(\cdot) \widehat{\otimes}_{A} X: \bmod -A \rightarrow \mathrm{LCS}^{\wedge}$ is exact;

(v) the functor $(\cdot) \widehat{\otimes}_{A} X: \bmod -A(\operatorname{Ban}) \rightarrow$ Ban is exact.

Proof. (i) $\Longleftrightarrow$ (ii) $\Longleftrightarrow$ (iii): this is Theorem 7.2.

(ii) $\Longrightarrow$ (iv) $\Longrightarrow(\mathrm{v})$ : this is clear.

(v) $\Longrightarrow$ (i). The proof is similar to the proof of implication (iii) $\Longrightarrow$ (i) in Theorem 7.2; one uses the equivalence of (i) and (iii) in Theorem 1.1.

Corollary 9.2. Let $A$ be a Fréchet-Arens-Michael algebra and $I \subset A_{+}$a closed left ideal. Set $X=A_{+} / I$. Suppose that there exists an Arens-Michael decomposition $A=$ $\varliminf_{\longleftarrow}\left(A_{\lambda}, \sigma_{\mu}^{\lambda}\right)_{\lambda \in \mathbb{N}}$ such that for each $\lambda \in \mathbb{N}$ the ideal $I_{\lambda}=\overline{\left(\sigma_{\lambda}\right)_{+}(I)}$ is weakly complemented in $\left(A_{\lambda}\right)_{+}$. Then the following conditions are equivalent:

(i) I has a right locally b.a.i.;

(ii) $X$ is a strictly flat Fréchet $A$-module;

(iii) $X$ is a flat Fréchet $A$-module.

Remark 9.3. Like Theorem 7.2 and Corollary 7.3, Theorem 9.1 and Corollary 9.2 have obvious "unital" versions. Specifically, if $A$ is unital, then we can replace $A_{+}$by $A$, "mod" by "unmod", and "mod" by "unmod" everywhere in the statements of Theorem 9.1 and Corollary 9.2. 
Remark 9.4. The additional condition imposed on $I_{\lambda}$ in Theorem 9.1 and in Corollary 9.2 looks rather unnatural, but we do not know how to put it into a more reasonable form. In particular, it is not clear whether or not this condition depends on the particular choice of an Arens-Michael decomposition. Also we do not know which relation (if any) this condition has to the position of $I$ inside $A_{+}$. Fortunately, as we shall see in Theorem 9.7 below, there is an important situation where this condition is satisfied automatically.

Let us recall some notation from [20, VII.2]. Let $A$ be an Arens-Michael algebra, and let $A^{e}=A_{+} \widehat{\otimes} A_{+}^{\text {op }}$ be the enveloping algebra of $A$. Denote by $\pi: A^{e} \rightarrow A_{+}$the product map, and set $I^{\Delta}=\operatorname{Ker} \pi$. Note that $I^{\Delta}$ is a closed left ideal of $A^{e}$, and that $A_{+}$is isomorphic to $A^{e} / I^{\Delta}$ in $A^{e}$-unmod $=A$-mod- $A$. Moreover, $I^{\Delta}$ is complemented in $A^{e}$, since the map $A_{+} \rightarrow A^{e}, a \mapsto 1_{+} \otimes a$ is readily seen to be a right inverse of $\pi$.

Similarly, set $A_{0}^{e}=A \widehat{\otimes} A^{\mathrm{op}}$, denote by $\pi_{0}: A_{0}^{e} \rightarrow A$ the product map, and set $I_{0}^{\Delta}=$ $\operatorname{Ker} \pi_{0}$. Finally, set $M=\operatorname{Ker}\left(\varepsilon_{A} \otimes \varepsilon_{A}\right) \subset A^{e}$. Note that $M=A_{+} \widehat{\otimes} A+A \widehat{\otimes} A_{+}$.

Now choose an Arens-Michael decomposition $A=\underset{\lim }{\longleftarrow}\left(A_{\lambda}, \sigma_{\mu}^{\lambda}\right)_{\lambda \in \Lambda}$. For each $\lambda \in \Lambda$, we use the symbols $A_{\lambda}^{e},\left(A_{0}^{e}\right)_{\lambda}, \pi_{\lambda},\left(\pi_{0}\right)_{\lambda}, I_{\lambda}^{\Delta},\left(I_{0}^{\Delta}\right)_{\lambda}, M_{\lambda}$ to denote the respective algebras, maps and ideals constructed from $A_{\lambda}$, as described above. For each $\lambda \prec \mu$, we set

$$
\begin{aligned}
\tau_{\lambda}^{\mu} & =\left(\sigma_{\lambda}^{\mu}\right)_{+} \otimes\left(\sigma_{\lambda}^{\mu}\right)_{+}: A_{\mu}^{e} \rightarrow A_{\lambda}^{e} \\
\tau_{\lambda} & =\left(\sigma_{\lambda}\right)_{+} \otimes\left(\sigma_{\lambda}\right)_{+}: A^{e} \rightarrow A_{\lambda}^{e} .
\end{aligned}
$$

Lemma 9.5. For each $\lambda \in \Lambda$, we have

$$
I_{\lambda}^{\Delta}=\overline{\tau_{\lambda}\left(I^{\Delta}\right)}, \quad M_{\lambda}=\overline{\tau_{\lambda}(M)},
$$

and there exist Arens-Michael decompositions

$$
\begin{array}{ll}
A^{e} \cong \lim _{\longleftarrow} A_{\lambda}^{e}, & A_{0}^{e} \cong \lim _{\longleftarrow}\left(A_{0}^{e}\right)_{\lambda}, \\
I^{\Delta} \cong \lim _{\varkappa} I_{\lambda}^{\Delta}, & M \cong \lim _{\longleftarrow} M_{\lambda},
\end{array}
$$

where the linking maps are the restrictions of $\tau_{\lambda}^{\mu}$ to the respective subalgebras of $A_{\mu}^{e}$. If, in addition, $A$ has a one-sided locally b.a.i., then

$$
\left(I_{0}^{\Delta}\right)_{\lambda}=\overline{\left(\sigma_{\lambda} \otimes \sigma_{\lambda}\right)\left(I_{0}^{\Delta}\right)}
$$

and there exists an Arens-Michael decomposition

$$
I_{0}^{\Delta} \cong \lim _{\longleftarrow}\left(I_{0}^{\Delta}\right)_{\lambda} \text {. }
$$

Proof. Since $M_{\lambda}=\left(A_{\lambda}\right)_{+} \widehat{\otimes} A_{\lambda}+A_{\lambda} \widehat{\otimes}\left(A_{\lambda}\right)_{+}$, the second formula in (17) is immediate from the fact that $\sigma_{\lambda}: A \rightarrow A_{\lambda}$ has dense range. The first formula in (17) is proved similarly, by using the fact that $I_{\lambda}^{\Delta}$ is the smallest closed left ideal of $A_{\lambda}^{e}$ containing all elements of the form $1_{+} \otimes a-a \otimes 1_{+}\left(a \in\left(A_{\lambda}\right)_{+}\right)$(see [20, VII.2.11]).

Now suppose that $A$ has a one-sided locally b.a.i. By Corollary 6.8, each $A_{\lambda}$ has a one-sided b.a.i., and so the product map $A_{\lambda} \widehat{\otimes}_{A_{\lambda}} A_{\lambda} \rightarrow A_{\lambda}$ is an isomorphism (see [20, II.3.12]). Therefore $\left(I_{0}^{\Delta}\right)_{\lambda}$ coincides with the kernel of the canonical map

$$
\hat{\pi}_{\lambda}: A_{\lambda} \widehat{\otimes} A_{\lambda} \rightarrow A_{\lambda} \widehat{\otimes} A_{A_{\lambda}} A_{\lambda}, \quad a \otimes b \mapsto a \otimes b .
$$

On the other hand, $\operatorname{Ker} \hat{\pi}_{\lambda}$ is equal to the closure of the linear span of all elements of 
the form $a b \otimes c-a \otimes b c\left(a, b, c \in A_{\lambda}\right)$; see [20, II.3]. Using the fact that $\sigma_{\lambda}: A \rightarrow A_{\lambda}$ has dense range, we obtain (20).

The isomorphisms (18) follow from the fact that projective tensor product commutes with reduced inverse limits $[\mathbf{3 0}, 41.6]$. Now (19) and (21) follow from (17), (18), and (20) (cf. also $[\mathbf{9}, 2.5 .6]$ ).

Lemma 9.6. Let $A$ be a Fréchet-Arens-Michael-algebra with a locally b.a.i. Then $A$ is amenable if and only if $A$ is biflat.

Proof. Let $A=\lim _{\leftarrow} A_{\lambda}$ be an Arens-Michael decomposition of $A$. Since $A$ has a locally b.a.i., Corollary $\overleftarrow{6.8}$ shows that each $A_{\lambda}$ has a b.a.i. By [20, Lemma VII.2.12 (V)], this implies that for each $\lambda \in \mathbb{N}$ the ideal $M_{\lambda}$ has a b.a.i. Applying Lemma 9.5 and Corollary 6.8, we see that $M$ has a locally b.a.i. By Corollary 7.3, $\mathbb{C}=A^{e} / M$ is a flat Fréchet $A^{e}$-module. Applying Proposition 4.10 to the sequence $0 \rightarrow A \rightarrow A_{+} \rightarrow \mathbb{C} \rightarrow 0$ in $A^{e}$-mod(Fr), we get the result.

The following result generalizes Helemskii and Sheinberg's Theorem 1.2 and gives a partial answer to a problem posed by Helemskii [22, Problem 11].

Theorem 9.7. Let $A$ be a Fréchet-Arens-Michael algebra. The following conditions are equivalent:

(i) A is amenable;

(ii) for each Banach algebra $B$ such that there exists a homomorphism $\varphi: A \rightarrow B$ with dense range, the algebra $B$ is amenable;

(iii) for each Arens-Michael decomposition $A=\lim _{\longleftarrow}\left(A_{\lambda}, \sigma_{\lambda}^{\mu}\right)_{\lambda \in \mathbb{N}}$, all the $A_{\lambda}$ 's are amenable Banach algebras;

(iv) A is isomorphic to the reduced inverse limit of a sequence of amenable Banach algebras;

(v) $A_{+}$is a strictly flat Fréchet A-bimodule;

(vi) $A$ is biflat and has a locally b.a.i.;

(vii) $I^{\Delta}$ has a right locally b.a.i.;

(viii) A has a locally b.a.i., and $I_{0}^{\Delta}$ has a right locally b.a.i.

If, in addition, $A$ is quasinormable, then the above conditions are equivalent to the following:

(ix) $A$ is biflat and has a b.a.i.;

(x) $I^{\Delta}$ has a right b.a.i.;

(xi) A has a b.a.i., and $I_{0}^{\Delta}$ has a right b.a.i.

Proof. (i) $\Longrightarrow$ (ii). This is a special case of Proposition 4.14 (see also Remark 4.15).

(ii) $\Longrightarrow($ iii $) \Longrightarrow($ iv). This is clear.

(iv) $\Longrightarrow$ (vii). Let $A=\lim _{\longleftarrow}\left(A_{\lambda}, \sigma_{\lambda}^{\mu}\right)_{\lambda \in \mathbb{N}}$ be a reduced inverse limit of amenable Banach algebras. In what follows, we use the notation introduced before Lemma 9.5. By Helemskii and Sheinberg's Theorem 1.2, each $I_{\lambda}^{\Delta}$ has a right b.a.i., which is equivalent to (vii) by Lemma 9.5 and Corollary 6.8 , 
(vii) $\Longleftrightarrow$ (v) $\Longleftrightarrow$ (i). By Lemma 9.5, for each $\lambda \in \mathbb{N}$ we have $I_{\lambda}^{\Delta}=\overline{\tau_{\lambda}\left(I^{\Delta}\right)}$, so that $\overline{\tau_{\lambda}\left(I^{\Delta}\right)}$ is complemented in $A_{\lambda}^{e}$. Now the equivalence of (vii), (v), and (i) follows from Corollary 9.2 (cf. also Remark 9.3) applied to $A^{e}$ and $I^{\Delta}$.

(iv) $\Longleftrightarrow$ (viii). This is immediate from Helemskii and Sheinberg's Theorem 1.2, Corollary 6.8, and Lemma 9.5.

(i) \&(viii) $\Longrightarrow$ (vi) $\Longrightarrow$ (i). This readily follows from Lemma 9.6.

Now assume that $A$ is quasinormable and satisfies conditions (i)-(viii). Since quasinormability is inherited by projective tensor products $[\mathbf{2 5}, 15.6 .5]$ and by quotients (and a fortiori by complemented subspaces), we see that $A_{0}^{e}, A^{e}$, and $I^{\Delta}$ are quasinormable. Together with Theorem 8.4, this gives (ix) and (x). Arguing as in [20, Lemma VII.2.12 (III)], we see that (ix) and (x) together imply (xi).

As an application of the above theorem, we can characterize amenable FréchetArens-Michael algebras in terms of derivations.

Theorem 9.8. Let $A$ be a Fréchet-Arens-Michael algebra. The following conditions are equivalent:

(i) A is amenable;

(ii) for each Banach A-bimodule $X$, every continuous derivation from $A$ to $X^{*}$ is inner;

(iii) for each Fréchet $A$-bimodule $X$, every continuous derivation from $A$ to $X^{*}$ is inner.

Proof. (i) $\Longrightarrow$ (iii). Let $X$ be a Fréchet $A$-bimodule. Since $A_{+}$is amenable, the augmented complex (8) is exact. Therefore the dual complex of (8) is also exact (see, e.g., $[\mathbf{3 5}, 26.4])$. In particular, the short sequence

$$
C_{0}(A, X)^{*} \rightarrow C_{1}(A, X)^{*} \rightarrow C_{2}(A, X)^{*}
$$

is exact. Using the same argument as in the Banach algebra case (see [20]) and taking into account Proposition 3.2, we can identify (22) with the sequence

$$
X^{*} \stackrel{\delta^{0}}{\longrightarrow} \mathscr{L}\left(A, X^{*}\right) \stackrel{\delta^{1}}{\longrightarrow} \mathscr{B} i l\left(A \times A, X^{*}\right),
$$

where

$$
\left(\delta^{0} f\right)(a)=a \cdot f-f \cdot a, \quad\left(\delta^{1} \varphi\right)(a, b)=a \cdot \varphi(b)-\varphi(a b)+\varphi(a) \cdot b .
$$

Obviously, the kernel of $\delta^{1}$ is exactly the set of all continuous derivations from $A$ to $X^{*}$, while the image of $\delta^{0}$ is exactly the set of all inner derivations from $A$ to $X^{*}$. Since (23) is exact, this completes the proof of (iii).

(iii) $\Longrightarrow$ (ii). This is clear.

(ii) $\Longrightarrow$ (i). Let $A=\lim A_{\lambda}$ be an Arens-Michael decomposition of $A$. By Theorem 9.7, it suffices to show that $A_{\lambda}$ is amenable for each $\lambda$. Let $X$ be a Banach $A_{\lambda}$-bimodule, and let $D: A_{\lambda} \rightarrow X^{*}$ be a continuous derivation. Denoting by $\sigma_{\lambda}$ the canonical map of $A$ to $A_{\lambda}$, we see that $D \sigma_{\lambda}: A \rightarrow X^{*}$ is a continuous derivation. By (ii), $D \sigma_{\lambda}$ is inner. Since $\sigma_{\lambda}$ has dense range, it follows that $D$ is also inner. Thus $A_{\lambda}$ is amenable. In view of the above remarks, this completes the proof of (i).

Let us now turn to Johnson's Theorem 1.3. 
Theorem 9.9. Let $A$ be a Fréchet-Arens-Michael algebra. The following conditions are equivalent:

(i) $A$ is amenable;

(ii) A has a locally bounded approximate diagonal.

If, in addition, $A$ is quasinormable, then the above conditions are equivalent to the following:

(iii) A has a bounded approximate diagonal;

(iv) A has a virtual diagonal.

Proof. (i) $\Longleftrightarrow$ (ii). This is immediate from Theorem 9.7 and Corollary 6.16.

Now assume that $A$ is amenable and quasinormable, so that it satisfies condition (xi) of Theorem 9.7. Let $\left(e_{\alpha}\right)_{\alpha \in \Lambda}$ be a b.a.i. in $A$, and let $\left(u_{\beta}\right)_{\beta \in \Lambda^{\prime}}$ be a right b.a.i. in $I_{0}^{\Delta}$. Define an order on $\Lambda \times \Lambda^{\prime}$ by $(\alpha, \beta) \prec(\gamma, \delta)$ if and only if $\alpha \prec \gamma$ and $\beta \prec \delta$. For each $(\alpha, \beta) \in \Lambda \times \Lambda^{\prime}$, set $M_{\alpha \beta}=e_{\alpha} \otimes e_{\alpha}-u_{\beta}$. We claim that $\left(M_{\alpha \beta}\right)$ is a bounded approximate diagonal for $A$. Indeed, it is clear that $\left\{M_{\alpha \beta}\right\}$ is bounded. Next, for each $a \in A$ we have

$$
\left[a, M_{\alpha \beta}\right]=v\left(e_{\alpha} \otimes e_{\alpha}-u_{\beta}\right),
$$

where $v=a \otimes 1-1 \otimes a \in I_{0}^{\Delta}$, the product being taken in $A_{0}^{e}$. We have $v u_{\beta} \rightarrow v$, as $\left(u_{\beta}\right)$ is a right a.i. in $I_{0}^{\Delta}$. On the other hand, an easy computation (cf. [20, VII.2.12], $[6,2.9 .21],[8,8.1],[39,5.1 .4]$ for the Banach algebra case) shows that $\left(e_{\alpha} \otimes e_{\alpha}\right)$ is a b.a.i. in $A_{0}^{e}$. Together with (24), this implies that $\left[a, M_{\alpha \beta}\right] \rightarrow 0$ for each $a \in A$.

Furthermore, we have $\pi_{0}\left(M_{\alpha \beta}\right)=e_{\alpha}^{2}$, and it is easily seen that $\left(e_{\alpha}^{2}\right)$ is a b.a.i. in $A$. Indeed, for each continuous submultiplicative seminorm $\|\cdot\|$ on $A$ we have

$$
\left\|e_{\alpha}^{2} a-a\right\| \leqslant\left\|e_{\alpha}^{2} a-e_{\alpha} a\right\|+\left\|e_{\alpha} a-a\right\| \leqslant\left\|e_{\alpha} a-a\right\|\left(\left\|e_{\alpha}\right\|+1\right) \rightarrow 0,
$$

as $\left(e_{\alpha}\right)$ is bounded. Thus $\pi_{0}\left(M_{\alpha \beta}\right) a \rightarrow a$ for each $a \in A$, and so $\left(M_{\alpha \beta}\right)$ is a bounded approximate diagonal for $A$.

(iii) $\Longleftrightarrow$ (iv). This follows from Lemma 5.7.

Recall that a complete topological algebra $A$ with involution is a locally $C^{*}$-algebra if the topology of $A$ can be defined by a family of $C^{*}$-seminorms. A $\sigma-C^{*}$-algebra (or a Fréchet $C^{*}$-algebra) is a metrizable locally $C^{*}$-algebra. Each locally $C^{*}$-algebra is an Arens-Michael algebra. Moreover, for each continuous $C^{*}$-seminorm $p$ on $A$, the Banach algebra $A_{p}$ is a $C^{*}$-algebra in a natural way, so that $A$ is a reduced inverse limit of $C^{*}$-algebras. For details, see [11].

A locally $C^{*}$-algebra $A$ is nuclear [41] if for each continuous $C^{*}$-seminorm $p$ on $A$ the $C^{*}$-algebra $A_{p}$ is nuclear. Equivalently, $A$ is nuclear if and only if $A$ is a reduced inverse limit of nuclear $C^{*}$-algebras $[4,4.1]$.

Combining equivalence (i) $\Longleftrightarrow$ (ii) from Theorem 9.7 with the Connes-Haagerup Theorem (see $[\mathbf{5 0}, 6.5 .12]$ ), we get the following.

Corollary 9.10. A $\sigma-C^{*}$-algebra is amenable if and only if it is nuclear.

Following [34], we say that a Hausdorff topological algebra $A$ is uniform if the topology on $A$ can be defined by a directed family $\left\{\|\cdot\|_{\lambda}: \lambda \in \Lambda\right\}$ of submultiplicative seminorms satisfying $\left\|a^{2}\right\|_{\lambda}=\|a\|_{\lambda}^{2}$ for each $a \in A$. Each uniform algebra is commutative and semisimple [34]. 
Recall that a Hausdorff topological space $X$ is hemicompact if the family of compact subsets of $X$ has a countable cofinal subset. A Hausdorff topological space $X$ is a $k$ space if a subset $F \subset X$ is closed whenever $F \cap K$ is closed for every compact subset $K \subset X$.

Given a commutative locally convex algebra $A$, we denote by $\Omega(A)$ the spectrum of $A$ (i.e., the space of nonzero continuous characters) endowed with the Gel'fand topology. In what follows, the algebra $C(X)$ of continuous functions on a topological space $X$ will be endowed with the compact-open topology.

Corollary 9.11. Let $A$ be a unital uniform Fréchet algebra. The following conditions are equivalent:

(i) $A$ is amenable;

(ii) $A$ is topologically isomorphic to $C(X)$ for a hemicompact $k$-space $X$;

(iii) the Gel'fand transform $\Gamma_{A}: A \rightarrow C(\Omega(A))$ is a topological isomorphism.

Proof. (i) $\Longrightarrow$ (iii). By $[\mathbf{1 3}, 4.1 .3], \Gamma_{A}$ is a topological isomorphism onto its image, so we may identify $A$ with $\Gamma_{A}(A)$. By $[\mathbf{1 3}, 3.2 .8], X=\Omega(A)$ is hemicompact, so that there exists a countable exhaustion $X=\bigcup_{n} K_{n}$ with $K_{n}$ compact such that each compact subset of $X$ is contained in some $K_{n}$. Without loss of generality, we assume that $K_{n} \subset K_{n+1}$ for every $n$. Let $A_{n}$ denote the uniform closure of the subalgebra $\left\{\left.f\right|_{K_{n}}: f \in A\right\}$ of $C\left(K_{n}\right)$. By [13, 4.1.6], the canonical map $A \rightarrow \lim A_{n}$ is a topological isomorphism. Using Theorem 9.7, we see that $A_{n}$ is amenable for each $n$. By Sheinberg's Theorem [53] (see also $[\mathbf{6}, 5.6 .2]$ ), $A_{n}=C\left(K_{n}\right)$. Thus we see that the composition of the maps

$$
A \hookrightarrow C(X) \rightarrow \lim _{\longleftarrow} C\left(K_{n}\right)
$$

is an isomorphism. Since the second map is obviously injective, we conclude that $A=C(X)$, as required.

(iii) $\Longrightarrow$ (ii). This follows from $[\mathbf{1 3}, 3.1 .9$ (iii), Theorem].

(ii) $\Longrightarrow$ (i). Let $X=\bigcup_{n} K_{n}$ be a countable exhaustion of $X$ with compact sets having the above property. Then $C(X) \cong \lim C\left(K_{n}\right)$ is an Arens-Michael decomposition of $C(X)[\mathbf{1 3}, 4.1 .7]$. Since each $C\left(K_{n}\right)$ is amenable (see, e.g., [20, VII.2.40], [6, 5.6.2]), Theorem 9.7 implies that $C(X)$ is amenable.

We end this section by giving two examples of amenable Fréchet-Arens-Michael algebras that are not covered by Corollaries 9.10 and 9.11 .

Example 9.12. Given a group $G$ and $g \in G$, let $\delta_{g}$ denote the function on $G$ which is 1 at $g, 0$ elsewhere. It is easy to see that for each group homomorphism $\varphi: G \rightarrow H$ we have a Banach algebra morphism $\varphi_{*}: \ell^{1}(G) \rightarrow \ell^{1}(H)$ uniquely determined by $\varphi_{*}\left(\delta_{g}\right)=\delta_{\varphi(g)}(g \in G)$. Moreover, if $\varphi$ is onto, then so is $\varphi_{*}$. Now let $\mathscr{G}=\left(G_{n}, \varphi_{n}^{m}\right)_{n \in \mathbb{N}}$ be an inverse sequence of groups such that the linking maps $\varphi_{n}^{m}$ are onto. Set $\mathscr{L}^{1}(\mathscr{G})=$ $\lim _{(}\left(\ell^{1}\left(G_{n}\right),\left(\varphi_{n}^{m}\right)_{*}\right)$. Clearly, $\mathscr{L}^{1}(\mathscr{G})$ is a Fréchet-Arens-Michael algebra. A standard argument shows that $\mathscr{L}^{1}(\mathscr{G})$ is not normable unless the sequence $\mathscr{G}$ stabilizes. Applying now Theorem 9.7 , we see that $\mathscr{L}^{1}(\mathscr{G})$ is amenable if and only if all the $G_{n}$ 's are amenable. 
Recall that a Fréchet space $E$ is a quojection if $E$ is isomorphic to a reduced inverse $\operatorname{limit} \lim \left(E_{n}, \varphi_{n}^{m}\right)$ of Banach spaces such that the linking maps $\varphi_{n}^{m}$ are onto. All $\sigma-C^{*}-$ algebras $[\mathbf{1 1}, 10.24]$ and all algebras of the form $\mathscr{L}^{1}(\mathscr{G})$ are quojections. We now give an example of an amenable Fréchet-Arens-Michael algebra which is not a quojection.

Example 9.13. Let $G$ be an infinite locally compact group, and let $A_{p}(G)(1<p<\infty)$ denote the Figà-Talamanca-Herz algebra on $G$ (see, e.g., $[\mathbf{6}, 4.5]$ ). The norm on $A_{p}(G)$ will be denoted by $\|\cdot\|_{p}$. Suppose that $G$ is amenable. Then, by [24], for each $p \geqslant q \geqslant 2$ we have $A_{q}(G) \subset A_{p}(G)$, and the inclusion of $A_{q}(G)$ into $A_{p}(G)$ is continuous with dense range. Moreover, as was shown in [5], $A_{p}(G) \neq A_{q}(G)$ if $p \neq q \geqslant 2$. Given $p \geqslant 2$, set $A_{p+}(G)=\bigcap_{q>p} A_{q}(G)=\lim _{q>p} A_{q}(G)$. Clearly, $A_{p+}(G)$ is a FréchetArens-Michael algebra. To show that $A_{p+}(G)$ is not a quojection, fix any $q>p$ and note that $\|\cdot\|_{q}$ is a continuous norm on $A_{p+}(G)$. By $[\mathbf{4 0}, 8.4 .33]$, a quojection having a continuous norm is normable. Therefore if $A_{p+}(G)$ were a quojection, the norms $\|\cdot\|_{q}$ and $\|\cdot\|_{r}$ on $A_{p+}(G)$ would be equivalent whenever $q, r$ are close enough to $p$, which contradicts the fact that the inclusion $A_{q}(G) \subset A_{r}(G)(2 \leqslant q<r)$ is proper.

Now assume that $G$ contains an abelian subgroup of finite index. Then $A_{2}(G)$ is amenable [31], and hence $A_{p}(G)$ is amenable for every $p$, since the inclusion $A_{2}(G) \rightarrow A_{p}(G)$ has dense range. Now Theorem 9.7 implies that $A_{p+}(G)$ is amenable.

\section{A counterexample}

Now we present an example of a commutative Fréchet-Arens-Michael algebra with a locally b.a.i., but without a b.a.i. Together with Corollary 7.3, this will show that Helemskii and Sheinberg's Theorem 1.1 does not extend verbatim to Fréchet-ArensMichael algebras.

Let $P$ be a family of real-valued sequences such that for each $i \in \mathbb{N}$ there exists $p \in P$ with $p_{i}>0$. Suppose also that $P$ is directed, i.e., for each $p, q \in P$ there exists $r \in P$ such that $r_{i} \geqslant \max \left\{p_{i}, q_{i}\right\}$ for all $i \in \mathbb{N}$. Recall that the Köthe sequence space $\lambda(P)$ is defined as follows:

$$
\lambda(P)=\left\{a=\left(a_{i}\right)_{i \in \mathbb{N}} \in \mathbb{C}^{\mathbb{N}}:\|a\|_{p}=\sum_{i}\left|a_{i}\right| p_{i}<\infty \forall p \in P\right\} .
$$

In the sequel, for each $i \in \mathbb{N}$ we set $e_{i}=(0, \ldots, 0,1,0, \ldots)$, where the single nonzero entry is in the $i$ th slot. The linear span of the $e_{i}$ 's is denoted by $c_{00}$.

Lemma 10.1. Suppose that $p_{i} \geqslant 1$ for each $i \in \mathbb{N}$. Then there exists a unique continuous product on $\lambda(P)$ such that $e_{i} e_{j}=e_{\min \{i, j\}}$ for all $i, j \in \mathbb{N}$. Together with this product, $\lambda(P)$ becomes a commutative Arens-Michael algebra.

Proof. Clearly, the map $\left(e_{i}, e_{j}\right) \mapsto e_{\min \{i, j\}}$ uniquely extends to an associative product on $c_{00}$. Since $p_{i} \geqslant 1$, we have $\left\|e_{i} e_{j}\right\|_{p} \leqslant\left\|e_{i}\right\|_{p}\left\|e_{j}\right\|_{p}$ for all $i, j \in \mathbb{N}$. This easily implies that $\|a b\|_{p} \leqslant\|a\|_{p}\|b\|_{p}$ for all $a, b \in c_{00}$. Using the density of $c_{00}$ in $\lambda(P)$ and extending the product by continuity to the whole of $\lambda(P)$, we get the result.

The Arens-Michael algebra defined in Lemma 10.1 will be denoted by $A(P)$. Note that for each $p \in P$ the Banach algebra $A(P)_{p}$ is isometrically isomorphic to $A(\{p\})$.

Lemma 10.2. The algebra $A(P)$ has no identity. 
Proof. Assume, towards a contradiction, that $u=\left(u_{i}\right)$ is an identity in $A(P)$. Then for each $j \in \mathbb{N}$ we have

$$
e_{j}=e_{j} u=\sum_{i} u_{i} e_{j} e_{i}=\sum_{i<j} u_{i} e_{i}+\left(\sum_{i \geqslant j} u_{i}\right) e_{j} .
$$

This implies that $u_{i}=0$ for all $i<j$. As $j \in \mathbb{N}$ was arbitrary, we obtain $u=0$, which is a contradiction.

Lemma 10.3. Suppose that each sequence $p \in P$ has a bounded subsequence. Then $A(P)$ has a locally b.a.i.

Proof. Let $p \in P$, and let $\left(p_{n_{k}}\right)_{k \in \mathbb{N}}$ be a bounded subsequence of $p$. Clearly, for each $a \in c_{00}$ we have $a e_{n_{k}}=a$ for $k$ large enough. Since $\left\|e_{n_{k}}\right\|=p_{n_{k}}$, this implies that $e_{n_{k}}$ is a b.a.i. in $\left(c_{00},\|\cdot\|_{p}\right)$ and hence in $A(\{p\})(c f .[8,1.4])$. Now the result follows from Corollary 6.8.

Now we define $P$ exactly as in the classical Köthe-Grothendieck example $[\mathbf{1 4}, \mathbf{2 9}]$. Namely, for each $k \in \mathbb{N}$ we define an infinite matrix $\alpha^{(k)}=\left(\alpha_{i j}^{(k)}\right)_{i, j \in \mathbb{N}}$ by setting

$$
\alpha_{i j}^{(k)}= \begin{cases}j^{k}, & i<k \\ i^{k}, & i \geqslant k .\end{cases}
$$

Fix a bijection $\varphi: \mathbb{N}^{2} \rightarrow \mathbb{N}$ such that $\varphi(i, j+1)<\varphi(i, j)$ for $i, j \in \mathbb{N}$. For each $k \in \mathbb{N}$, define a sequence $p^{(k)}=\left(p_{n}^{(k)}\right)_{n \in \mathbb{N}}$ by $p_{n}^{(k)}=\alpha_{\varphi^{-1}(n)}^{(k)}$. Finally, set $P=\left\{p^{(k)}: k \in \mathbb{N}\right\}$. Since $P$ is countable, $A(P)$ is a Fréchet algebra.

Proposition 10.4. If $P$ is as above, then $A(P)$ has a locally b.a.i., but does not have a b.a.i.

Proof. Given $p=p^{(k)} \in P$, set $n_{i}=\varphi(k, i)(i \in \mathbb{N})$. By assumption, the sequence $\left(n_{i}\right)$ is strictly increasing. We have $p_{n_{i}}^{(k)}=\alpha_{k i}^{(k)}=k^{k}$, so that $\left(p_{n_{i}}^{(k)}\right)_{i \in \mathbb{N}}$ is a bounded subsequence of $p^{(k)}$. By Lemma 10.3, $A(P)$ has a locally b.a.i. On the other hand, $A(P)$ is a Montel space $[\mathbf{1 4}, \mathbf{2 9}]$. Applying Lemma 5.1 and Lemma 10.2, we see that $A(P)$ does not have a b.a.i.

Together with Corollary 7.3 this gives the following.

Corollary 10.5. There exists a commutative Fréchet-Arens-Michael algebra A such that the trivial Fréchet $A$-module $\mathbb{C}=A_{+} / A$ is strictly flat, but $A$ does not have a b.a.i. In addition, the underlying Fréchet space of $A$ is Montel.

\section{Acknowledgements}

The author is grateful to A.Ya. Helemskii for helpful discussions.

\section{References}

[1] G.R. Allan, Stable inverse-limit sequences, with application to Fréchet algebras, Studia Math. 121 (1996), no. 1, 277-308. 
[2] G.R. Allan, Stable inverse-limit sequences and automatic continuity, Studia Math. 141 (2000), no. 2, 99-107.

[3] R. Arens, A generalization of normed rings, Pacific J. Math. 2 (1952), 455-471.

[4] S.J. Bhatt and D.J. Karia, Complete positivity, tensor products and $C^{*}$ nuclearity for inverse limits of $C^{*}$-algebras, Proc. Indian Acad. Sci. Math. Sci. 101 (1991), no. 3, 149-167.

[5] M.G. Cowling and J.J.F. Fournier, Inclusion and noninclusion of spaces of convolution operators, Trans. Amer. Math. Soc. 221 (1976), no. 1, 59-95.

[6] H.G. Dales, Banach algebras and automatic continuity, Clarendon Press, Oxford, 2000.

[7] H.G. Dales, R.J. Loy and Y. Zhang, Approximate amenability for Banach sequence algebras, Studia Math. 177 (2006), no. 1, 81-96.

[8] R.S. Doran and J. Wichmann, Approximate identities and factorization in Banach modules, Lecture Notes in Math. 768, Springer-Verlag, New York, 1979.

[9] R. Engelking, General topology, PWN, Warsaw, 1977.

[10] J. Eschmeier and M. Putinar, Spectral decompositions and analytic sheaves, Clarendon Press, Oxford, 1996.

[11] M. Fragoulopoulou, Topological algebras with involution, North-Holland Mathematics Studies 200, Elsevier, Amsterdam, 2005.

[12] F. Ghahramani and R.J. Loy, Generalized notions of amenability, J. Funct. Anal. 208 (2004), no. 1, 229-260.

[13] H. Goldmann, Uniform Fréchet algebras, North-Holland Mathematics Studies 162, North-Holland, Amsterdam, 1990.

[14] A. Grothendieck, Sur les espaces $(F)$ et $(D F)$, Summa Brasil. Math. 3 (1954), 57-123.

[15] A. Guichardet, Sur l'homologie et la cohomologie des algèbres de Banach, C. R. Acad. Sci. Paris Ser. A 262 (1966), 38-41.

[16] S.L. Gulick, The bidual of a locally multiplicatively-convex algebra, Pacific J. Math. 17 (1966), 71-96.

[17] A.Ya. Helemskii, A periodic product of modules over Banach algebras (Russian), Funktsional. Anal. i Prilozhen. 5 (1971), no. 1, 95-96; English transl.: Functional Anal. Appl. 5 (1971), 84-85.

[18] A.Ya. Helemskii and M.V. Sheinberg, Amenable Banach algebras (Russian), Funktsional. Anal. i Prilozhen. 13 (1979), no. 1, 42-48; English transl.: Functional Anal. Appl. 13 (1979), no. 1, 32-37.

[19] A.Ya. Helemskii, Flat Banach modules and amenable algebras (Russian), Trudy Moskov. Mat. Obshch. 47 (1984), 179-218; English transl.: Trans. Moscow Math. Soc. (1985), 199-244.

[20] A.Ya. Helemskii, The homology of Banach and topological algebras, Moscow University Press, 1986 (Russian); English transl.: Math. Appl. (Soviet Ser.) 41, Kluwer Academic Publishers, Dordrecht, 1989. 
[21] A.Ya. Helemskii, Banach and polynormed algebras: general theory, representations, homology, Nauka, Moscow, 1989 (Russian); English transl.: Oxford University Press, New York, 1993.

[22] A.Ya. Helemskii, 31 problems of the homology of the algebras of analysis, in Linear and complex analysis: Problem book 3, Part I (eds. V.P. Havin and N.K. Nikolski), Lecture Notes in Math. 1573, 54-78, Springer-Verlag, New York, 1994.

[23] A.Ya. Helemskii, Homology for the algebras of analysis, Handbook of algebra, Vol. 2 (ed. M. Hazewinkel), 151-274, North-Holland, Amsterdam, 2000.

[24] C. Herz, The theory of $p$-spaces with an application to convolution operators, Trans. Amer. Math. Soc. 154 (1971), 69-82.

[25] H. Jarchow, Locally convex spaces, Teubner, Stuttgart, 1981.

[26] B.E. Johnson, An introduction to the theory of centralizers, Proc. London Math. Soc. (3) 14 (1964), 299-320.

[27] B.E. Johnson, Cohomology in Banach algebras, Mem. Amer. Math. Soc. 127, A.M.S., Providence, RI (1972).

[28] B.E. Johnson, Approximate diagonals and cohomology of certain annihilator Banach algebras, Amer. J. Math. 94 (1972). no. 3, 685-698.

[29] G. Köthe, Die Stufenräume, eine einfache Klasse linearer vollkommener Räume, Math. Z. 51 (1948), 317-345.

[30] G. Köthe, Topological vector spaces II, Springer-Verlag, New York, 1979.

[31] A.T.-M. Lau, R.J. Loy and G.A. Willis, Amenability of Banach and $C^{*}$-algebras on locally compact groups, Studia Math. 119 (1996), no. 2, 161-178.

[32] S. MacLane, Homology, Springer-Verlag, New York, 1963.

[33] S. MacLane, Categories for the working mathematician, Grad. Texts in Math. Vol. 5. Springer-Verlag, New York, 1971.

[34] A. Mallios, Topological algebras. Selected topics, North-Holland Mathematics Studies 124, North-Holland, Amsterdam, 1986.

[35] R. Meise and D. Vogt, Introduction to functional analysis, Clarendon Press, Oxford, 1997.

[36] E.A. Michael, Locally multiplicatively-convex topological algebras, Mem. Amer. Math. Soc. 11, A.M.S., Providence, RI (1952).

[37] V.P. Palamodov, The projective limit functor in the category of topological linear spaces (Russian), Mat. Sb. (N.S.) 75 (1968), 567-603; English transl.: Math. USSR-Sb. 75 (1968), 529-558.

[38] V.P. Palamodov, Homological methods in the theory of locally convex spaces (Russian), Uspehi Mat. Nauk 26 (1971), no. 1 (157), 3-65; English transl.: Russian Math. Surveys 26 (1971), 1-64.

[39] T.W. Palmer, Banach algebras and the general theory of *-algebras. Vol. I, Algebras and Banach algebras, Encycl. of Math. and its Appl. 49, Cambridge Univ. Press, Cambridge, 1994.

[40] P. Pérez Carreras and J. Bonet, Barrelled locally convex spaces, North-Holland Mathematics Studies 131, North-Holland, Amsterdam, 1987. 
[41] N.C. Phillips, Inverse limits of $C^{*}$-algebras, J. Operator Theory 19 (1988), no. 1 , 159-195.

[42] A.Yu. Pirkovskii, On the problem of the existence of a sufficient number of injective Fréchet modules over nonnormable Fréchet algebras (Russian), Izvestiya Ross. Akad. Nauk, ser. matem. 62 (1998), no. 4, 137-154; English transl.: Izvestiya: Mathematics 62 (1998), no. 4, 773-788.

[43] A.Yu. Pirkovskii, On Arens-Michael algebras which do not have non-zero injective $\widehat{\otimes}$-modules, Studia Math. 133 (1999), no. 2, 163-174.

[44] A.Yu. Pirkovskii, Injective topological modules, additivity formulas for homological dimensions and related topics, Topological homology: Helemskii's Moscow seminar (ed. A. Ya. Helemskii), 93-143, Nova Sci. Publ., Huntington, NY, 2000.

[45] A.Yu. Pirkovskii, Strictly flat cyclic Fréchet modules and approximate identities, Topological algebras and applications, 359-366, Contemp. Math. 427, Amer. Math. Soc., Providence, RI, 2007.

[46] A.Yu. Pirkovskii, Arens-Michael envelopes, homological epimorphisms, and relatively quasi-free algebras (Russian), Tr. Mosk. Mat. Obs. 69 (2008), 34-123; English transl.: Trans. Moscow Math. Soc. (2008), 27-104.

[47] C.P. Podara, On strictly flat Fréchet modules, Topological algebras and applications, 389-399, Contemp. Math. 427, Amer. Math. Soc., Providence, RI, 2007.

[48] F. Prosmans, Derived categories for functional analysis, Publ. Res. Inst. Math. Sci. 36 (2000), no. 1, 19-83.

[49] D. Quillen, Higher algebraic $K$-theory I: Higher $K$-theories, Lecture Notes in Math. 341, 85-147, Springer-Verlag, New York, 1973.

[50] V. Runde, Lectures on amenability, Lecture Notes in Math. 1774, SpringerVerlag, New York, 2002.

[51] H. Schaefer, Topological vector spaces, The Macmillan Co., New York, 1966.

[52] Yu.V. Selivanov, Cohomological characterizations of biprojective and biflat Banach algebras, Monatsh. Math. 128 (1999), no. 1, 35-60.

[53] M.V. Sheinberg, A characterization of the algebra $C(\Omega)$ in terms of cohomology groups (Russian), Uspekhi Mat. Nauk 32 (1977), no. 5, 203-204.

[54] B. Stenström, Rings of quotients, Die Grundlehren der Mathematischen Wissenschaften, Band 217, Springer-Verlag, New York, 1975.

[55] J.L. Taylor, Homology and cohomology for topological algebras, Adv. Math. 9 (1972), 137-182.

[56] J. Wengenroth, Derived functors in functional analysis, Lecture Notes in Math. 1810, Springer-Verlag, New York, 2003.

A.Yu. Pirkovskii pirkosha@sci.pfu.edu.ru, pirkosha@online.ru

Department of Nonlinear Analysis and Optimization, Faculty of Science, Peoples' Friendship University of Russia, Mikluho-Maklaya 6, 117198 Moscow, Russia 